Linköping Studies in Science and Technology. Dissertations, No. 1550

\title{
Mathematical Optimization of HDR Brachytherapy
}

\section{Åsa Holm}

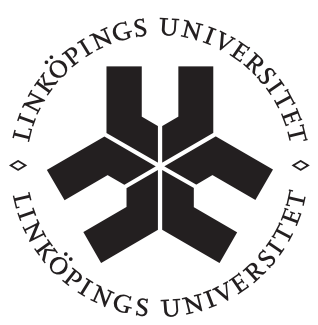

\section{Linköping University}

\section{INSTITUTE OF TECHNOLOGY}

Department of Mathematics

Linköping University

SE-581 83 Linköping, Sweden

Linköping 2013 
Linköping Studies in Science and Technology. Dissertations,

No. 1550

\title{
Mathematical Optimization of HDR Brachytherapy
}

\author{
asa.holmeliu.se \\ www.mai.liu.se \\ Division of Optimization \\ Department of Mathematics \\ Linköping University \\ SE-581 83 Linköping \\ Sweden
}

ISBN 978-91-7519-496-7

ISSN 0345-7524

Printed by LiU-Tryck, Linköping, Sweden 2013 


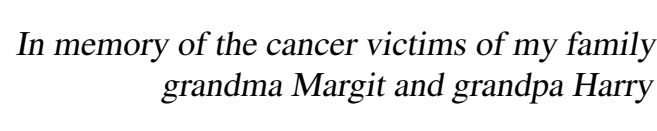





\section{Abstract}

One out of eight deaths throughout the world is due to cancer. Developing new treatments and improving existing treatments is hence of major importance. In this thesis we have studied how mathematical optimization can be used to improve an existing treatment method: high-dose-rate (HDR) brachytherapy.

HDR brachytherapy is a radiation modality used to treat tumours of for example the cervix, prostate, breasts, and skin. In HDR brachytherapy catheters are implanted into or close to the tumour volume. A radioactive source is moved through the catheters, and by adjusting where the catheters are placed, called catheter positioning, and how the source is moved through the catheters, called the dwelling time pattern, the dose distribution can be controlled.

By constructing an individualized catheter positioning and dwelling time pattern, called dose plan, based on each patient's anatomy, it is possible to improve the treatment result. Mathematical optimization has during the last decade been used to aid in creating individualized dose plans. The dominating optimization model for this purpose is a linear penalty model. This model only considers the dwelling time pattern within already implanted catheters, and minimizes a weighted deviation from dose intervals prescribed by a physician.

In this thesis we show that the distribution of the basic variables in the linear penalty model implies that only dwelling time patterns that have certain characteristics can be optimal. These characteristics cause troublesome inhomogeneities in the plans, and although various measures for mitigating these are already available, it is of fundamental interest to understand their cause.

We have also shown that the relationship between the objective function of the linear penalty model and the measures commonly used for evaluating the quality of the dose distribution is weak. This implies that even if the model is solved to optimality there is no guarantee that the generated plan is optimal with respect to clinically relevant objectives, or even near-optimal. We have therefore constructed a new model for optimizing the dwelling time pattern. This model approximates the quality measures by the concept conditional value-at-risk, and we show that the relationship between our new model and the quality measures is strong. Furthermore, the new model generates dwelling time patterns that yield high-quality dose distributions.

Combining optimization of the dwelling time pattern with optimization of the catheter positioning yields a problem for which it is rarely possible to find a proven optimal solution within a reasonable time frame. We have therefore developed a variable neighbourhood search heuristic that outperforms a state-of-the-art optimization software (CPLEX). We have also developed a tailored branch-and-bound algorithm that is better at improving the dual bound than a general branch-and-bound algorithm. This is a step towards the development of a method that can find proven optimal solutions to the combined problem within a reasonable time frame. 



\section{Populärvetenskaplig sammanfattning}

Varje år diagnostiseras cirka 55000 personer i Sverige med cancer. Vilken typ av behandling de får beror till exempel på typen av cancer. En möjlig behandling är brachyterapi, vilket är en typ av strålbehandling. I den här avhandlingen har vi använt optimeringsmetodiker för att ta fram matematiska metoder och modeller som förbättrar planeringen av behandlingen, vilket kan ge större chans för överlevnad och mindre risk för biverkningar.

Brachyterapi är en form av strålbehandling där strålkällan placeras i eller mycket nära cancertumören. Målet med behandlingen är att ge en tillräckligt hög, det vill säga dödlig, dos till tumören samtidigt som frisk vävnad skadas så lite som möjligt. För att planera brachyterapi krävs många olika steg, men ett av de viktigaste är att bestämma var strålkällorna placeras och hur länge de stannar i varje position. Dessa beslut avgör nämligen hur dosfördelningen ser ut, det vill säga hur dosen till tumör och omgivande organ ser ut.

Olika dosfördelningar kan jämföras med ett antal kvantitativa mått som är korrelerade med sannolikheten att döda cancern och risken för biverkningar. Detta innebär att man utifrån dessa mått kan avgöra vilken av alla möjliga dosplaner som är den bästa. Optimeringslära handlar om just detta, hur man på ett effektivt sätt skall hitta den bästa lösningen bland ett mycket stort antal möjliga lösningar till ett beslutsproblem.

Det fanns tidigare en optimeringsmodell för dosplaneringsproblemet och vi presenterar i denna avhandling några brister med den gamla modellen. Vi har visat att modellen har en egenskap som leder till att få strålpositioner kan väljas och därför måste några av bestrålningstiderna bli mycket långa. Dessa långa bestrålningstider har också noterats $\mathrm{i}$ praktiken och anses vara ett problem eftersom de orsakar oönskade biverkningar. Vi har också studerat hur väl den tidigare modellen optimerar med avseende på de kvantitativa måtten, och våra studier visar att modellen enbart bristfälligt tar hänsyn till dessa. Detta tyder på att den tidigare modellen egentligen löser fel problem, det vill säga hittar den bästa lösningen med avseende på ett felaktigt mått.

I denna avhandling har vi därför tagit fram en ny optimeringsmodell som väl beskriver alla möjliga dosfördelningar och kopplar dem med de kvantitativa måtten som beskriver dosfördelningars egenskaper. Vi har visat att vår nya modell inte har de brister som den tidigare modellen hade. Vi har också utvecklat en metod som effektivt hittar optimum till modellen, det vill säga den bästa möjliga dosfördelningen. Med hjälp av denna modell och metod kan behandlingen snabbt och enkelt anpassa till varje persons unika anatomi, och patienten behandlas med den dosfördelning som är optimal för dem. Detta ger stora tidsbesparingar för personalen och förhoppningsvis även större chans för överlevnad för patienten och mindre risk för biverkningar. 



\section{Acknowledgments}

Doing a PhD is hard. There are many steps that you need to figure out along the way, and without the help of others it is almost impossible to reach the final goal: a dissertation. Having reached this goal I would like to thank some of those that helped along the way.

Torbjörn Larsson, my main supervisor, for sharing his wisdom and knowledge. Åsa Carlsson Tedgren, my co-supervisor, for introducing me to the field of brachytherapy, and for always trying to understand what I've been doing even though it is far from her field. Also the research school in interdisciplinary mathematics deserves a thank you for giving me the opportunity to work in my favorite field; how to apply optimization to real world problems.

The doctors and radiophysicists, especially Håkan Hedtjärn and Peter Larsson, for helping me along the way by providing data, answering questions, and showing me around. I especially appreciate your genuine interest, now when the work is done, in the results and how it can be applied in the clinic. Your work now is equally important for making my research useful.

All my present and former colleagues at the Department of Mathematics, for always being interested in discussing a problem and sharing your knowledge. A special thank you to the $\mathrm{PhD}$-students, present and former, for sharing your experiences, it's always easier when you know that you are not alone.

Mikael Call, my office roommate during these five years, for helping me with everything from how bibtex works to discussing mathematical proofs. You have been invaluable. Elina Rönnberg, for always taking the time to ask, and listen, to what is going on. I wish I could see the world through your eyes sometimes.

Susanne Gennow and Paul Vaderlind, for opening my eyes to the wonderful world of mathematics. Maud Göthe-Lundgren, for showing how optimization use and develop some of the best parts of mathematics.

All of my friends, for making sure that even when work or life is hard I have something to look forward to. Nothing is as good a cure as laughing over some good food and a board game.

My wonderful family, for your never ending support. I doubt that you had any idea what you where getting into many years ago when you told me "Of course you should apply".

Lastly Rolle, there is no way that I would have been able to complete this dissertation without you picking me up when all I wanted to do was quit. You have been an invaluable support, and without you my life would be less, I love you.

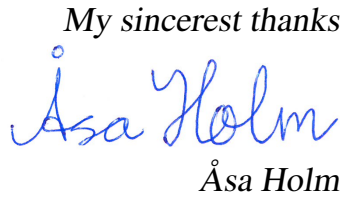

Linköping, October 18, 2013 



\section{Contents}

1 Introduction 1

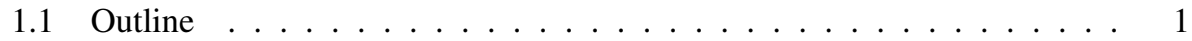

1.1.1 Outline of Part I . . . . . . . . . . . . . . . . 2

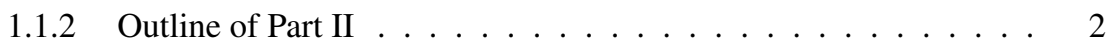

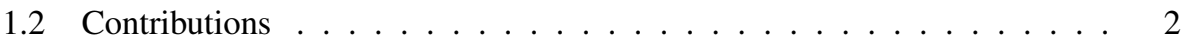

1.2.1 Main contributions of the appended papers . . . . . . . . 2

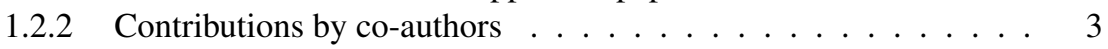

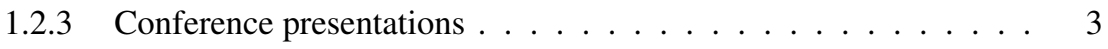

I Radiotherapy and Optimization 5

2 Cancer and Radiotherapy $\quad 7$

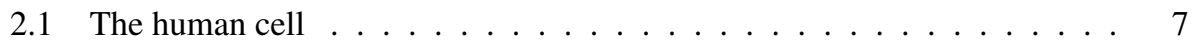

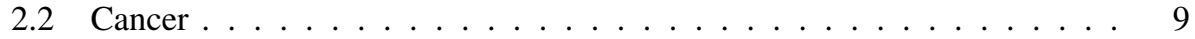

2.3 Radiation . . . . . . . . . . . . . . . . . 10

2.3.1 Particle radiation . . . . . . . . . . . . . . . 10

2.3.2 Electromagnetic radiation . . . . . . . . . . . . . . 11

2.3 .3 Sources of radiation . . . . . . . . . . . . . . . . 12

2.4 Biological impact of radiation $\ldots \ldots \ldots \ldots$

2.4.1 How radiation damages the cell . . . . . . . . . . . . . 13

2.4.2 Measuring biological effect of radiation . . . . . . . . . . . 14

2.4.3 Biological impact of radiation on tissue . . . . . . . . . 15

2.5 Radiotherapy . . . . . . . . . . . . . . . . . . 15

2.5.1 Fractionated radiotherapy $\ldots \ldots \ldots \ldots \ldots$

2.5.2 External beam radiotherapy . . . . . . . . . . . . . . 17

2.5 .3 Brachytherapy . . . . . . . . . . . . . . 18 
2.5.4 HDR brachytherapy for prostate cancer . . . . . . . . . . 20

2.5.5 Treatment plans . . . . . . . . . . . . . . 22

2.5.6 Evaluation of dose plans . . . . . . . . . . . 23

2.5.7 Dosimetric protocols . . . . . . . . . . . . 26

3 Optimization of Radiotherapy 29

3.1 Problem formulation . . . . . . . . . . . . . . . . . . . . . . . 29

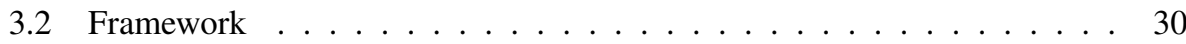

3.2.1 Dose point generation . . . . . . . . . . . . 30

3.2 .2 Dose calculations . . . . . . . . . . . . . . 31

3.3 Earlier models and research . . . . . . . . . . . . . . . 32

3.3 .1 Subproblems . . . . . . . . . . . . . . 32

3.3.2 Penalty models for dwell time distribution optimization . . . . . . 33

3.3.3 Dwell time distribution optimization models that include evaluation measures . . . . . . . . . . . . 35

3.3.4 Optimization of catheter positioning . . . . . . . . . 38

4 Contributions of the Thesis 41

4.1 Properties of the linear penalty model . . . . . . . . . . . . . . . 41

4.2 Alternative models for optimizing the dwell time distribution . . . . . . . . 42

4.2.1 An alternative penalty model . . . . . . . . . . . . . 42

4.2.2 A model including dosimetric indices . . . . . . . . . . . . . 44

4.2.3 Theoretical comparison of the linear penalty model and our dosimetric model . . . . . . . . . . . . . . . 51

4.3 Methods for optimization of the catheter positioning . . . . . . . . . 53

4.3.1 Using commercial software .............. 53

4.3 .2 Heuristics . . . . . . . . . . . . . . . 54

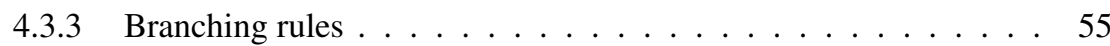

4.4 Future research . . . . . . . . . . . . . . . . . 57

Bibliography

II Appended Papers $\quad 65$

A Impact of Using Linear Optimization Models in Dose Planning for HDR $\begin{array}{ll}\text { Brachytherapy } & 67\end{array}$

1 Introduction . . . . . . . . . . . . . . . . . 70

2 Mathematical model and theory ............... 71

$2.1 \quad$ Analysis of the linear penalty model . . . . . . . . . . 72

$2.2 \quad$ Alternative penalty . . . . . . . . . . . . . 75

3 Materials and methods . . . . . . . . . . . . . . . . . . 77

$4 \quad$ Results . . . . . . . . . . . . . . . . . . . . 78

5 Summary and conclusion . . . . . . . . . . . . . . 81

References . . . . . . . . . . . . . . . . . . . 82 
B Study of the Relationship Between Dosimetric Indices and Linear Penalties in Dose Distribution Optimization for HDR Prostate Brachytherapy 85

1 Introduction . . . . . . . . . . . . . . . . . 88

2 Methods and material . . . . . . . . . . . . . . . . . . 89

3 Results . . . . . . . . . . . . . . . . . . . . . . . . . . . . . . . 90

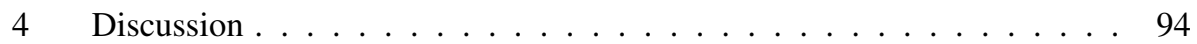

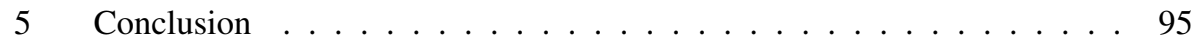

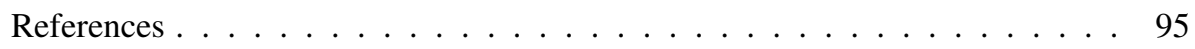

C A Linear Programming Model for Optimizing HDR Brachytherapy Dose Distributions with Respect to Mean Dose in the DVH-tail 97

1 Introduction . . . . . . . . . . . . . . . . . . 100

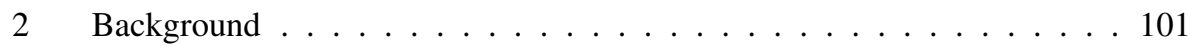

2.1 Dose calculations . . . . . . . . . . . . . . 101

2.2 Dosimetric indices . . . . . . . . . . . . . . 101

2.3 Conditional value-at-risk . . . . . . . . . . . . . . . . 104

3 Mathematical formulation . . . . . . . . . . . . . . . . . 105

3.1 Constraints on CVaR . . . . . . . . . . . . . . 106

3.2 Maximizing homogeneity . . . . . . . . . . . . 107

3.3 Mathematical model . . . . . . . . . . . . . . . . 108

3.4 Comparison with other models . . . . . . . . . . . . . . 109

4 Methods and materials . . . . . . . . . . . . . . 110

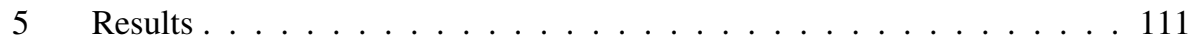

6 Summary and conclusion . . . . . . . . . . . . . . . . 114

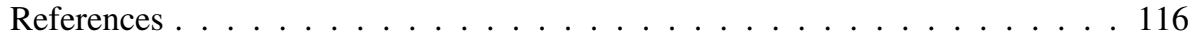

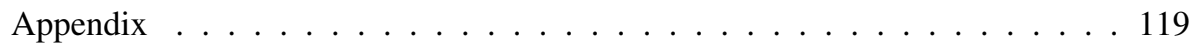

D Heuristics for Integrated Optimization of Catheter Positioning and Dwell $\begin{array}{lr}\text { Time Distribution in Prostate HDR Brachytherapy } & 121\end{array}$

1 Introduction . . . . . . . . . . . . . . . . . . . . . . 124

2 Problem description . . . . . . . . . . . . . . . . 125

3 Mathematical models . . . . . . . . . . . . . . . . . . . . 127

3.1 Original model for the DTDOP . . . . . . . . . . . . 127

3.2 Alternative model for the DTDOP . . . . . . . . . . . . 128

3.3 Integrating catheter placement with DTDOP . . . . . . . . . 129

4 Heuristics for CLP and CPLP . . . . . . . . . . . . . . . . 130

4.1 The neighbourhood and its characteristics . . . . . . . . . 131

4.2 Tabu search . . . . . . . . . . . . . . . . . 132

4.3 Variable neighbourhood search . . . . . . . . . . . . . . 134

4.4 Genetic algorithm . . . . . . . . . . . . . . . . . . . . . . . . . . . . . . . . . . . . . .

5 Computational studies . . . . . . . . . . . . . . . 137

$5.1 \quad$ Dataset . . . . . . . . . . . . . . . 137

5.2 Results and discussion . . . . . . . . . . . . . . . . . . . . . . . . . . . . . . . . .

6 Conclusion and future research . . . . . . . . . . . . . . . . . . . 144

References ......................... 146 
E A Tailored Branch-and-Bound Method for Optimizing the Dwelling Time Pattern and Catheter Positioning in HDR Brachytherapy

1 Introduction . . . . . . . . . . . . . . . . . . . . . . . 152

2 Optimization of HDR brachytherapy . . . . . . . . . . . . . . . . 152

3 Branch-and-bound . . . . . . . . . . . . . . . . 154

4 Our tailored branch-and-bound . . . . . . . . . . . . . . . . 157

4.1 The relaxation . . . . . . . . . . . . . 157

4.2 The branching rule . . . . . . . . . . . . . . . . 158

4.3 Solving the subproblems . . . . . . . . . . . . . . 159

5 Computational studies . . . . . . . . . . . . . . . . . 159

$5.1 \quad$ Test setup . . . . . . . . . . . . . . . . . . 159

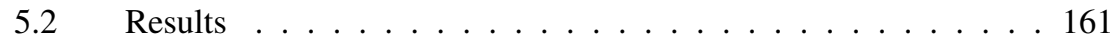

6 Discussion and conclusion . . . . . . . . . . . . . . . . . 164

References . . . . . . . . . . . . . . . . 165 


\section{Introduction}

This thesis is concerned with the problem of how to generate high quality dose plans for high dose-rate (HDR) brachytherapy. HDR brachytherapy is one type of radiotherapy, and it is used to treat several sorts of cancer such as tumours of the cervix, oesophagus, lungs, breasts, skin and prostate. As with all types of treatment, planning is needed before treatment can actually commence. One of the planning steps is to create a dose plan that for example decides how the radiation should be delivered.

During the last decade, the use of mathematical optimization as an aid when creating dose plans for HDR brachytherapy has increased. To facilitate optimization of HDR brachytherapy research has of course been needed, and still is, to create suitable models and methods for solving them. Our research within this field is focused one three areas: properties of the mathematical optimization model that is most frequently used in clinical practice, new optimization models for finding superior dose plans, and methods for solving such optimization models that have been extended to also include a decision that has not been optimized before. We have in our studies focused on the case when brachytherapy is applied to prostate cancer, but most of the results should be applicable also for other treatment sites.

\subsection{Outline}

This thesis consists of this introduction and two other parts. The introduction includes one section that summarizes the main contributions of the thesis, and a section that clarifies what my contribution is, and what is due to my co-authors. The outline of the other two parts are as follows. 


\subsubsection{Outline of Part I}

Applying optimization to real world problems often require a thorough understanding of the application under consideration. Chapter 2 therefore introduces fundamental issues related to HDR brachytherapy, such as why and how the treatment works, what planning that is needed, and how to evaluate dose plans. This chapter does not assume previous knowledge about cancer, radiation or radiotherapy. Readers that already have this knowledge can skip Chapter 2. Readers that are not interested in understanding more than is absolutely necessary about the treatment can skip most of Chapter 2, except Sections 2.5.3, 2.5.4 and 2.5.6. For those that would like to learn more we recommend the books 'Strålbehandling' by Degerfält et al. ${ }^{16}$ and 'Medicinsk fysik' by Berglund and Jönsson ${ }^{8}$ for a general introduction to radiotherapy. For those interested in radiobiology, the book 'Basic clinical radiobiology' by Joiner and van der Kogel ${ }^{26}$ provides an in-depth description.

Chapter 3 introduces the reader to optimization in radiotherapy. First a general problem description is given, and then the framework needed for performing the optimization is described. The chapter ends with a literature review of previous research within the field of optimization of HDR brachytherapy.

Part I ends with Chapter 4 that briefly describes the contributions of the appended papers in Part II. Chapter 4 also presents some other, minor, contributions that are not included in the papers. Lastly, the chapter ends with some suggestions on future research within the field.

\subsubsection{Outline of Part II}

Part II consists of five appended papers. The first two papers, Paper A and Paper B, present some properties of the optimization model most commonly used in clinical practice. Paper $\mathrm{C}$ presents a new optimization model that better corresponds to the goals of the dose distribution. The last two papers, Paper D and Paper E, consider methods for solving an optimization model that include more of the decisions of the dose plan than previous models.

\subsection{Contributions}

This section describes the contributions of the thesis, and of the co-authors.

\subsubsection{Main contributions of the appended papers}

The main contributions of the appended research papers are:

\section{Paper A - Impact of Using Linear Optimization Models in Dose Planning for HDR Brachytherapy}

Shows that certain properties of the optimization model most commonly used in clinical practice are the cause of the dose plan inhomogeneities that physicians find troublesome. 
Paper B - Study of the Relationship Between Dosimetric Indices and Linear Penalties in Dose Distribution Optimization for HDR Prostate Brachytherapy

Shows that the relationship between the objective function of the optimization model most commonly used in clinical practice and the dosimetric indices used for evaluating the quality of a dose plans is inadequate, when applied to optimization of HDR prostate brachytherapy.

Paper C - A Linear Programming Model for Optimizing HDR Brachytherapy Dose Distributions with Respect to Mean Dose in the DVH-tail

Presents a new model that correspond well with the dosimetric indices used for evaluation of dose plan quality.

Paper D - Heuristics for Integrated Optimization of Catheter Positioning and Dwell Time Distribution in Prostate HDR Brachytherapy

Presents a well functioning variable neighbourhood search heuristic for solving a catheter positioning extension of the optimization model most commonly used in clinical practice.

Paper E - A Tailored Branch-and-Bound Method for Optimizing the Dwelling Time Pattern and Catheter Positioning in HDR Brachytherapy

Develops a tailored branch-and-bound method for solving the model of Paper C, extended to also include catheter positioning.

\section{Publication status}

Paper A - has been published in Medical Physics.

Paper B - is submitted for publication.

Paper C - has been published in Medical Physics.

Paper D - will be published in Annals of Operations Research.

Paper E - has been printed as a technical report.

\subsubsection{Contributions by co-authors}

All papers except Paper E are co-authored with Torbjörn Larsson and Åsa Carlsson Tedgren. Their roles have been to supervise the work, and to some degree suggest directions of the research. The focus of Åsa Carlsson Tedgren has been the application, and Torbjörn Larsson has focused on the mathematical parts.

\subsubsection{Conference presentations}

During my $\mathrm{PhD}$ studies I have attended the following conferences.

Sixth Nordic Optimization Symposium, Göteborg, Sweden, 2013. I presented parts of Paper C. 
The 21st International Symposium on Mathematical Programming, Berlin, Germany, 2012. I presented parts of Paper B and C.

Operational Research Peripatetic Postgraduate Program, EURO conference for young OR researchers, Linz, Austria, 2012. I presented parts of Paper D. An earlier version of Paper D was also included in the conference proceeding of the conference.

Informs 2010 Annual Meeting, Austin, Texas, USA, 2010. I presented paper A.

Åsa Carlsson Tedgren has also presented my posters at two conferences which I was not able to attend.

The $2^{\text {nd }}$ ESTRO Forum, Geneva, Switzerland, 2013. The poster described parts of Paper C.

AAPM $55^{\text {th }}$ Annual Meeting and Exhibition, Indianapolis, Indiana, USA, 2013. The poster described parts of Paper C. 
Part I

Radiotherapy and Optimization 



\section{2}

\section{Cancer and Radiotherapy}

Radiotherapy is one kind of treatment used for cancer. To understand how optimization can be used to improve the treatment an understanding of both the disease and the treatment is needed. This chapter will provide a comprehensive introduction into this field. The organisation of the chapter is as follows: it starts by describing the human cell and cancer, thereafter the concept of radiation is introduced, continuing with the effect radiation has on cells and tissue, and ending with a description of different types of radiotherapy.

\subsection{The human cell}

Humans are made up of tissue, which in turn consists of eukaryotic cells (we will from now on refer to eukaryotic cells as only cells), and in each human there are approximately $10^{14}$ cells $^{9}$. The cells are responsible for all processes in the human body. The cell consists of many different components and one of these is the nucleus, where all DNA (deoxyribonucleic acid) is located. The DNA contains the genetic instructions used for the development and functioning of the cell and in human cells it is divided into several linear bundles called chromosomes.

DNA consists of two polynucleotide strands wrapped around each other to form a double helix. Each strand includes nucleobases. These bases are the genetic instructions and they are paired with bases on the other strand by hydrogen bonds. The base pairing is complementary, which in essence means that if you know one of the bases in the pair you automatically know the other as well. An illustration of DNA is shown in Figure 2.1. ${ }^{11}$

During a cell's life it goes through a number of different phases, and these phases make up the cell cycle. The phases are:

$G_{0}$ (Gap 0) This is a resting phase were the cell has, temporarily or permanently, left the cell cycle. No cell division occurs and the cell tends to its normal duties. 


\section{Polynucleotide chain}

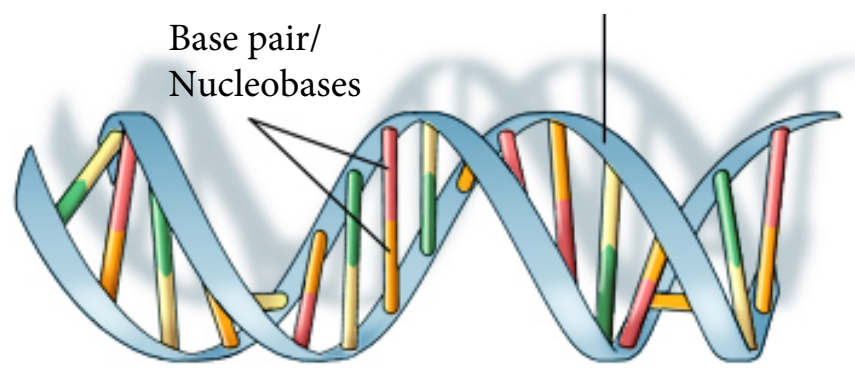

Figure 2.1: Illustration of the DNA, which is shaped as a double helix.

$G_{1}$ (Gap 1) The cell grows and prepares for synthesis. At the end of this phase, just prior to the $S$ phase, there is a checkpoint. At this checkpoint the cell ensures that it is ready for synthesis, especially the DNA is checked for errors. If any damages are detected the cell either tries to repair the damages or undergoes apoptosis (programmed cell death).

S (Synthesis) During this phase the DNA is duplicated, this is called replication.

$G_{2}$ (Gap 2) The cell continue to grow. At the end of this phase just before mitosis starts there is another DNA error checkpoint.

M (Mitosis) This is the phase where the cell is divided into two new cells. Mitosis is a very complicated process consisting of several subphases as well as a checkpoint.

In Figure 2.2 an illustration of the stages of the cell cycle is given. ${ }^{9}$

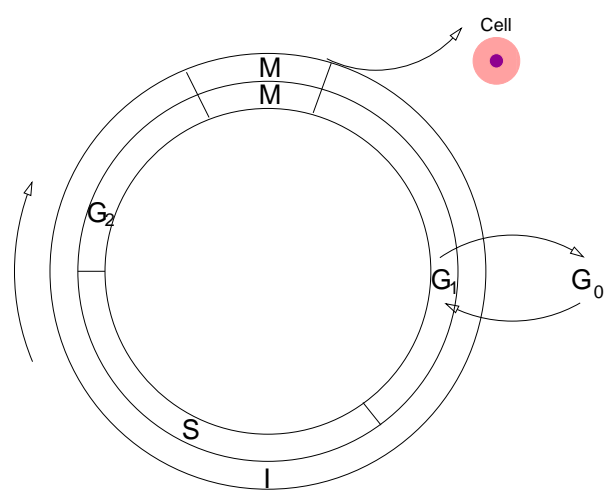

Figure 2.2: Illustration of stages of the cell cycle. 


\subsection{Cancer}

Cancer is apart from heart diseases the most common cause of death throughout the world. For the year of 2007 there were estimates of 12 million new cancer cases and around 7.6 million deaths related to cancer worldwide. This equals that that 1 out of 8 deaths is caused by cancer.

Cancer is not one disease but rather a group of diseases comprising over 200 different types, all characterised by uncontrolled growth and spread of abnormal cells ${ }^{19}$. The different types are usually named by the type of cell it starts in. Cancer diseases are caused by multiple changes (called mutations) in the DNA of a cell, changing the properties of that cell. Mutations can be caused by ionising radiation, tobacco etcetera, but most of them are spontaneous and occur frequently during the cell division in all types of cells. The difference with the mutations that cause cancer is that they give the cells a competitive advantage over their neighbouring cells, usually by changing the DNA encoding for proteins responsible for controlling the cell cycle. The properties the mutations need to cause in the cells are different for different types of cancers, but a number of key features can be distinguished:

- Cancer cells can reproduce without receiving growth-chemical signals that normal cells require.

- Cancer cells can continue to grow even though they receive "stop-growth" signals.

- Cancer cells are less likely to kill themselves when their DNA has been damaged.

- Cancer cells can influence the body to grow new blood vessels to supply the cancer cell with nutrients.

- Cancer cells can proliferate indefinitely unlike normal cells that can only divide a limited number of times.

- Cancer cells can break loose and travel to other parts of the body, this is called metastasis.

It is the last feature that makes cancer so lethal, out of 10 deaths in cancer, 9 are due to metastasis. $^{1,11}$

The choice of treatment type used for a patient with cancer does not only depend on the type of cancer the patient has, but also the extent of its spread, its sensitivity to treatment and factors related to the patients physical, psychological and social needs ${ }^{18}$. The most common treatment options are:

Surgery If the tumour is still localised it could be surgically removed. The goal is to remove all cancerous cells, this often require removing normal surrounding tissue and usually surrounding lymph nodes as well since cancer often metastasize through these.

Chemotherapy One property of cancerous cells is that they divide rapidly. In chemotherapy this is used by the delivery of drugs that target dividing cells, either causing them to undergo apoptosis or impairing mitosis. Since cancer cells are not the only cells dividing in the body side-effects are common, especially to cells that divide 
rapidly under normal circumstances such as bone marrow, digestive tract and hair follicles cells.

Radiotherapy Normal cells are better than cancerous cells at repairing damages to their DNA, and DNA damages in cells often lead to cell death or at least reduced cell reproduction. Radiotherapy uses these facts by trying to damage the DNA in the cancerous cells with ionising radiation.

Various treatments, such as the above-mentioned and other less common treatments, can be used either as a standalone therapy or combined in various ways.

\subsection{Radiation}

Radiation is a process where energy is transported through space either as electromagnetic waves (referred to as electromagnetic radiation) or as particles. One feature of radiation is that the energy radiates from its source, that is, the energy travels outwards in straight lines in all directions. Radiation is usually classified in one of two ways, either by how the energy is transported, as particle radiation or as electromagnetic radiation, or by the effect the radiation has on the irradiated medium. Below different types of radiation are described according to the first classification but first the reason for the second classification is presented.

The energy that is transported is deposited into the irradiated medium through interaction between the radiation and the medium. When the energy is transferred to the atoms of the irradiated medium, the electrons in the atoms may enter an excited state. This means that the electrons have gained energy and thereby moved to a higher orbit. If the energy is higher the result is more drastic and electrons may leave the atom, an illustration of this can be found in Figure 2.3. The process that causes electrons to leave the atoms is called ionisation. An atom that has lost an electron is positively charged, which significantly alters the characteristics of the atom compared to the neutral atom. Depending on the energy of the radiation the irradiated medium could become ionised, this is what is used for the second classification of radiation. Types of radiation that have high enough energy to cause ionisation is called ionising and if the energy is lower it is called non-ionising. Examples of ionising radiation are $\alpha$-particles, $\beta$-particles, X-rays and $\gamma$-rays (all of these are of interest when considering radiotherapy and are therefore presented in the following subsections), and examples of non-ionising radiation is radio waves and visible light.

\subsubsection{Particle radiation}

In particle radiation the energy is transported by subatomic particles, and the energy consists of the kinetic energy of the particle. Some common particles in this context are electrons, positrons, $\alpha$-particles, neutrons and protons. Below two of the common particles are described more, especially how they interact with the irradiated medium.

An $\alpha$-particle consists of two protons and two neutrons but no electrons, that is, $\alpha$ particles are helium nucleuses. They deposit their energy to a medium through collisions with the atoms of the medium, or rather mainly the electrons of the atoms. Through the collisions energy is transferred from the $\alpha$-particle to electrons in the media, but since the 

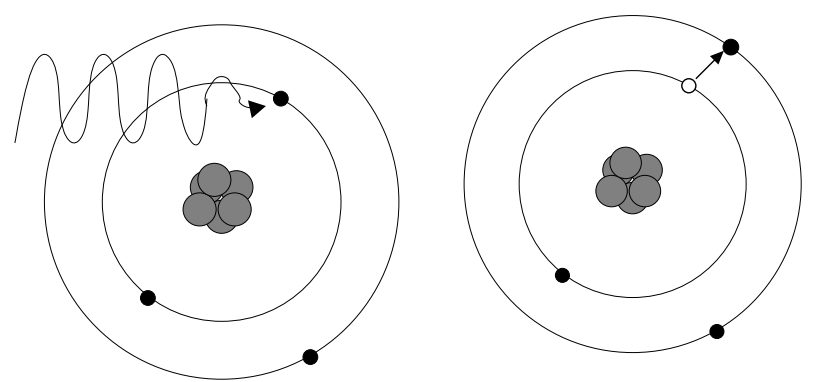

Radiation interacts with the atom The electron enters an excited state

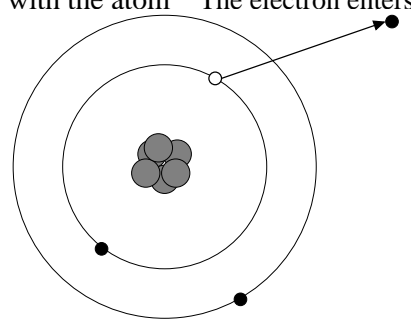

The electron leaves the atom, so the atom becomes ionized

Figure 2.3: Transfer of energy to an electron by radiation can cause the electron to enter an excited state or if the energy is high enough to leave the atom.

$\alpha$-particle is much heavier than electrons they only loss a small part of their energy and the collision does not change their direction significantly. This means that to deposit all of its energy it has to collide with many electrons, however since the $\alpha$-particle is quite big it will collide with all electrons that it passes and hence lose its energy quite quickly. As a result of this the $\alpha$-particles cause great damage along their path but also have a very short range, only less than a millimetre in tissue and they can be stopped by a single sheet of paper.

A $\beta$-particle is either an electron or a positron (that is, the electron's anti-particle). As with $\alpha$-particles they deposit their energy through collisions, the difference being that the $\beta$-particle is much lighter. This implies that their directions may change as a consequence of the collisions, and $\beta$-radiation does therefore have curved tracks. The range of $\beta$ particles is short, however much longer than $\alpha$-particles. In tissue the range is measured in millimetres and $\beta$-particles can be stopped be an aluminium plate.

\subsubsection{Electromagnetic radiation}

Electromagnetic radiation is a special form of energy that consists of electric and magnetic oscillations; it has no mass or kinetic energy in the strict sense. Two properties are important when considering electromagnetic radiation, frequency and wavelength. Wavelength is the spatial period of the wave, that is the length between two crests (see Figure 2.4). Frequency is the number of waves per time unit. There is a simple relationship between frequency $(f)$ and wavelength $(\lambda)$, namely $v=f \lambda$ where $v$ is the velocity of 
the radiation. Electromagnetic radiation also exhibits particle properties, especially when considering short times and distances, and is then referred to as photons. The photons are associated with waves with frequency proportional to the energy they carry, and the energy per photon is $E=h f$, where $h$ is Plank's constant.

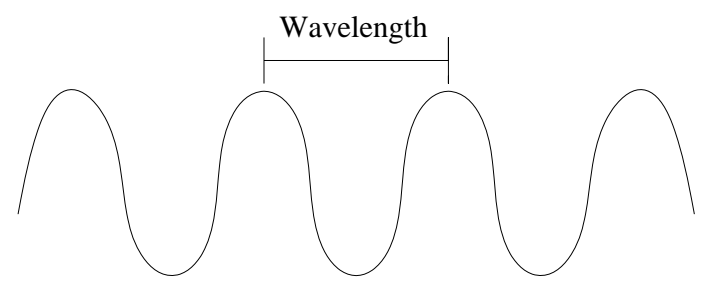

Figure 2.4: Illustration of wavelength.

Electromagnetic radiation is classified into different types depending on the frequency of the radiation (and thereby also the energy), examples of groups are radio waves, infrared radiation, visible light, $\mathrm{X}$-rays and $\gamma$-rays.

$\mathrm{X}$-rays and $\gamma$-rays are physically identical, the difference being only the origin. $\mathrm{X}$ rays are emitted by electrons outside the nucleus while $\gamma$-rays are emitted by the nucleus. The range of X-rays and $\gamma$-rays is long; it can reach several meters in tissue and hundreds of meters in air. To stop $\gamma$-rays or X-rays meter-thick concrete layers or decimetre-thick layers of lead are needed. The energy of X-rays and $\gamma$-rays can be from $12 \mathrm{eV}$ and up.

\subsubsection{Sources of radiation}

In radiotherapy there are mainly two types of sources for radiation that are used: radionuclides and accelerators. Below these two are described shortly.

\section{Radionuclides}

Isotopes are atoms that contain the same numbers of protons but a different number of neutrons. Unstable isotopes that undergo radioactive decay are called radionuclides. Radioactive decay means that the unstable isotopes send out one or more particles and/or electromagnetic radiation to get rid of excess energy (in rare cases it can also split into two approximately equal parts). There exist a number of different decay processes and the type and level of the energy emitted varies between radionuclides. The usual types are $\alpha$-particles, $\beta$-particles and $\gamma$-rays.

The decay processes follow a probabilistic behaviour, and this is the reason why not all atoms decay immediately and simultaneously. The probability of decay varies between radionuclides, causing different nuclides to decay at different rates. The period of time required for half of the radionuclides to decay is called half-life. The half-life is closely connected to the activity of a radionuclide, which is number of decays per second. 


\section{Accelerators}

A particle accelerator is a device that increases the speed of charged particles by using electromagnetic fields. In medicine accelerators are often used to create X-rays. This is done by accelerating electrons, produced by thermionic emission in the cathode, towards the anode, consisting of a metal with high melting temperature, by electric forces. Upon impact with the anode the electrons are rapidly decelerated, and as a result the kinetic energy is transform into other kinds of energy, mostly heat, but around one percent is emitted as X-rays. The energy of the X-rays depends on the kind of metal in the anode and the velocity and kinetic energy of the electrons. Depending of the intended use of the X-rays the mean photon energy is different, when used for diagnostic purposes such as mammography, dentistry and computed tomography it is in the range $20-250 \mathrm{kV}$, while the mean photon energy is in the range 3-10MV when used for radiotherapy.

Accelerators can also be used in radiotherapy to deliverer electron radiation, the energy of the electrons is then somewhere in the range $2-42 \mathrm{MV}$.

\subsection{Biological impact of radiation}

\subsubsection{How radiation damages the cell}

As described in Section 2.3 radiation deposits energy to the matter with which it interacts, which might cause ionisation. This might break or change the structure of the matter, all molecules in a cell can therefore be damaged by radiation. It has however been shown that the "target" in the cell most sensitive to ionising radiation is the DNA-helix. ${ }^{26} \mathrm{~A}$ disturbance in the combinations of the DNA string can yield devastating effects on the function of the cell. The damage imposed on the DNA by the radiation arises from two type of effects: the direct and the indirect effect. The most prominent effect depends on the type of radiation. For heavy particles, such as $\alpha$-particles, the direct effect is the most significant. For photon-radiation and $\beta$-particles on the other hand the indirect effect is the most significant. ${ }^{9}$

Damage due to the direct effects means that the radiation causes ionisation directly in the atoms of the DNA. The electrons that leave the atoms cause bonds to be broken, which can break the DNA strand. ${ }^{9}$

How damage due to the indirect effect occurs is more complicated. The radiation then interacts with the water in the cell, causing the water molecules to break and create free radicals. Radicals are highly reactive molecules, with unpaired electrons. Some of the radicals created, or products of the radicals, can travel through the cell to the nucleus where they act as a toxic for the DNA causing strands of the DNA to break. ${ }^{9}$

The breaks of the DNA strand can be of two types: single-strand break or doublestrand break. Here single-strand break means that only one of the strands of the DNAhelix is broken, and double-strand break means that both strands are broken (with less than 5 base pairs between the breaks). Damages such as breaks of the DNA can be repaired; however single-strand breaks are easier to repair since the other strand can be used as a template. Not all damages can be repaired and sometimes misrepair occurs; this can cause the cell to die. There are many factors that affect the cells' ability to repair DNA-damage, such as in which phase of the cell cycle the cell is when the damage occurs, the number 
of breaks, and the type of cell. The ability to repair damage is lower for tumour cells than for healthy tissue. ${ }^{9}$

\subsubsection{Measuring biological effect of radiation}

The impact of the radiation is hence not due to high energy levels as such but rather that the transferred engergy damages the wrong molecules ${ }^{8}$. There is however a connection between the energy absorbed and the probability of producing harmful effects. In radiotherapy a basic quantity used for measuring the absorbed energy is the absorbed dose. This is defined as the amount of imparted energy per unit mass of absorbent material and is measured in Gray (Gy). Below we will mean absorbed dose when we say dose. Another important concept in this context is dose-rate which is absorbed dose per time unit. ${ }^{16}$

To measure the connection between the dose and the probability of producing harmful effects, a common method is to observe cell survival after radiation with various doses. Curves showing survival against dose are called cell survival curves. If the dose is plotted on the $\mathrm{x}$-axis and survival on the y-axis a sigmoid curve is obtained (see Figure 2.5a), however it is more common to plot the logarithm of the survival on the y-axis and then a semilogaritmic curve is obtained (see Figure $2.5 \mathrm{~b}$ ). There are many models trying to describe the observed curve, one that is widespread in both experimental and clinical radiobiology is the linear-quadratic (LQ) model. In the LQ-model the formula describing cell survival probability $S$ (which is the same as surviving fraction) is $S=e^{-\alpha D-\beta D^{2}}$, where $\mathrm{D}$ is the dose and $\alpha$ and $\beta$ are parameters. ${ }^{26}$

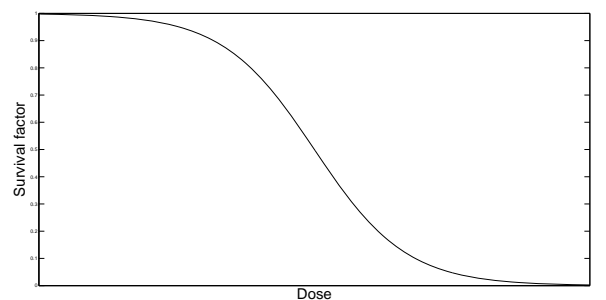

(a) Sigmoid curve

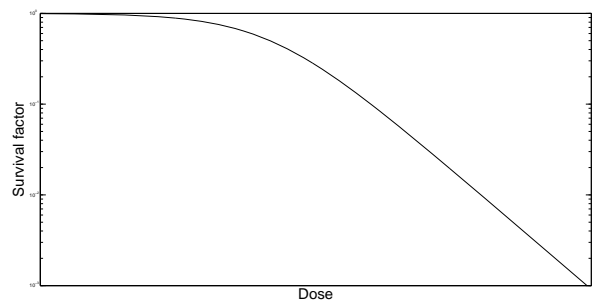

(b) Semilogaritmic curve

Figure 2.5: Cell survival curves.

There are many factors that affect the cell survival curve. Some of these are:

Cell cycle The cells' sensitivity to radiation varies throughout the cell cycle. It is most sensitive while in mitosis or late $G_{2}$, and most resistant in the $\mathrm{S}$ phase and $G_{0}$ phase. This is one reason for the success of radiotherapy as treatment for cancer, since tumour cells divide more rapidly the probability of cells being in mitosis is higher for tumour cells than for healthy cells.

Oxygen Lack of oxygen makes cells more resistant to radiation. The reason for this is that oxygen increases the toxicity of free radicals. Healthy cells are always well 
oxygenated while there are often tumour cells that have poor access to oxygen (cells that have poor access to oxygen are called hypoxic).

Dose-rate Using a lower dose-rate yields higher survival fraction. The reason for this is that all cells get more time to repair damages and proliferate.

Radiation type Radiosensitivity is higher for heavy particles such as $\alpha$-particles than for $\beta$-particles and photons, due to higher probability for double strand breaks. ${ }^{9}$

\subsubsection{Biological impact of radiation on tissue}

When using radiation as a treatment the interesting result is not the effect on each cell but rather how the tumour and surrounding healthy tissue respond. Dose-response curves show how the incidence of a radiation effect depends on dose. Examples of radiation effects could be cancer cure, or side-effects of different types. All dose-response curves have a sigmoid shape. The dose-response curve related to tumour cure (control) is often called tumour control (cure) probability (TCP). TCP can be mathematically modelled and several models exist, and one very common model defines TCP as:

$$
\begin{gathered}
T C P=\prod_{i=1}^{n} T C P\left(d_{i}, N_{i}\right), \\
T C P\left(d_{i}, N_{i}\right)=e^{N_{i} * S_{i}}=e^{N_{i} e^{-\alpha d_{i}-\beta d_{i}^{2}} .}
\end{gathered}
$$

Here the tumour volume is divided into $n$ subvolumes with $N_{i}$ tumour cells in subvolume $i$, and $d_{i}$ is the dose to subvolume $i$. As can be seen the surviving fraction of cells is used in this function and modelled by the LQ-model. ${ }^{9,26}$

Creating models for healthy tissue response is harder since many different side-effects can occur and there are different levels of severity of each side-effect. For this thesis we settle with noting that there exists models also for normal tissue response and that these yield a measure called normal tissue complication probability (NTCP). TCP and NTCP can be used to estimate the success of treatment regarding both cure and side-effects. ${ }^{9}$

The farther apart the curves for TCP and NTCP with respect to dose are, the better the chances for a good treatment result. In this context a concept called therapeutic ratio (TR) is often introduced. Therapeutic ratio is the relationship between tumour control dose and the tolerance dose for normal tissue, and should be as high as possible. The formula is TR=DNTCP/DTCP where DTCP is dose relative the likelihood of cure and DNTCP is dose relative the likelihood of side-effects. The concept of TR is illustrated in Figure 2.6. ${ }^{16}$

\subsection{Radiotherapy}

Radiotherapy is the use of ionising radiation as a treatment for cancer and a few other diseases. As described in Section 2.4 radiation damages cells in predicable ways, and cancer cells are for several reasons more sensitive to radiation than healthy cells. This is a fundamental reason that makes radiotherapy a viable choice.

Radiotherapy can be divided into three subgroups: 


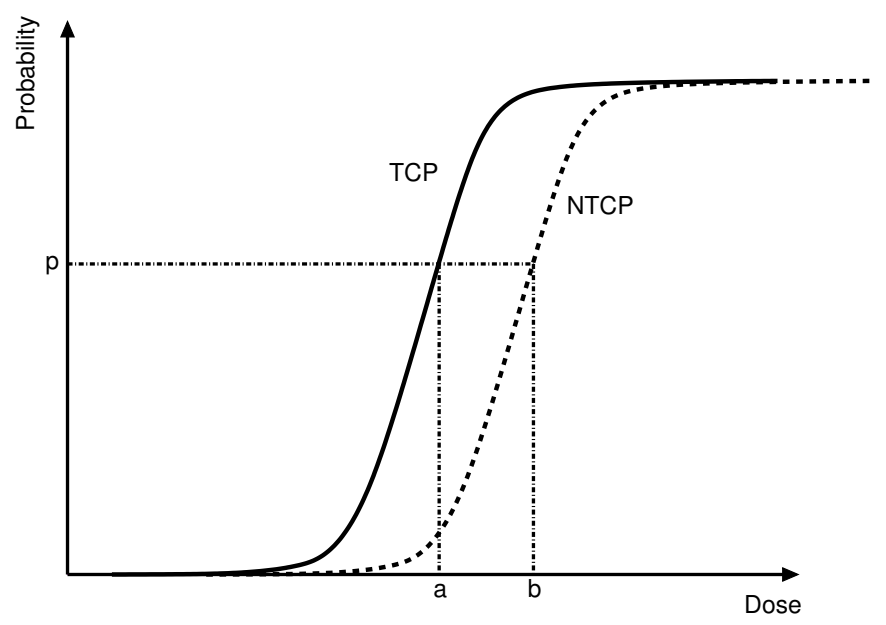

Figure 2.6: Illustration of therapeutic ratio (TR). For the probability $p$ for tumour control and impact on normal tissue the dose required is a and $b$, respectively. Hence $T R=a / b$.

External beam radiotherapy During external beam radiotherapy the radioactive source is localised outside the body. The most common source is a linear accelerator that generates electron or photon radiation, but protons and heavier ions are also used.

Brachytherapy During brachytherapy the radioactive source is localised inside or next to the volume requiring treatment. Brachy is a Greek word meaning "short-distance“.

Systemic radioisotope therapy During systemic radioisotope therapy radioisotopes that target specific cells are delivered through infusion (into the bloodstream) or ingestion. Targeting can be due to chemical properties of the isotope or by attaching the radioisotope to another molecule or antibody that guides it to the target tissue.

Of the three subgroups external beam therapy is the most common one. Some different external beam radiotherapy treatment types are described in more detail in Sections 2.5.2.

The portion of patients with cancer in Sweden that were given radiotherapy at some point during their treatment was $47 \%$ in 2001 . Approximately half of the radiotherapy treatments were given as a curative treatment, the rest as palliative treatment (where cure is not possible and the aim is pain relief or local disease control). ${ }^{53}$

Radiotherapy is in itself painless, however due to the damage of healthy cells sideeffects may occur. Most of the side-effects that occur are predictable and expected, and they are usually limited to the organs that receive radiation. The nature, severity, and longevity of side-effects depend on, among others, the treatment area, the type of radiation, the dose, and the patient. Organs that might suffer from side-effects due to radiation are referred to as organs-at-risk (OAR). 


\subsubsection{Fractionated radiotherapy}

As is well known radiotherapy is usually delivered in several fractions, that is, the dose is not delivered all at once but divided into fractions delivered with some time between them. There are four main reasons for this:

Repopulation Both normal cells and tumour cells proliferate even after radiation exposure. The increase in tumour cells does of course work against the treatment but the increase in normal cells instead reduce the risk of side-effects and is therefore in favour of the treatment.

Repair Since normal cells are better at repairing damage due to radiation than tumour cells, allowing enough time between fractions for normal cells to repair is in favour of the treatment.

Redistribution Since radiosensitivity varies throughout the cell cycle fractionated radiotherapy increases the probability of tumour cells being exposed to radiation during a sensitive phase. Since tumour cells divide more frequently than normal cells this will cause more damage to tumour cells than to normal cells, since mitosis is one of the most sensitive phases.

Reoxygenation As noted above hypoxic cells are more resistant to radiation than well oxygenated cells. When well oxygenated tumour cells die the hypoxic cells will become increasingly oxygenated thereby increasing their sensitivity to radiation. ${ }^{9}$

\subsubsection{External beam radiotherapy}

There are several types of external beam radiotherapy, and the same type can be called different things dependent on the manufacturer of the machines. Here we will describe some of these.

One of the most common types is called 3-dimensional conformal radiation therapy $(3 \mathrm{D}-\mathrm{CRT})^{36}$. In 3D-CRT radiation beams, of either photons or electrons, are formed by a linear accelerator and directed at the patient by a gantry. The difference compared to 2D-CRT which was common earlier, is that the planning is based on a 3D-reconstruction of the treatment volume instead of a 2D-reconstruction. Intensity-modulated radiation therapy (IMRT) is a specialized form of 3D-CRT that divides each beam into a number of beamlets using a multileaf collimator. The multileaf collimator can block parts of the radiation beam when it passes through the collimator, and by changing which parts that are blocked it is possible to differentiate the intensity of the beamlets. This allows the dose to conform better to the 3-D shape of the tumour. ${ }^{17}$ Recently a technique called VMAT (volumetric-modulated arc therapy) has been developed. VMAT can be seen as a specialized version of IMRT, where the gantry of the linear accelerator continuously rotates around the patient.

External beam radiotherapy can also use heavier particles than electrons, such as protons. Such treatments utilise that the energy deposed by an ionizing heavier particle increases as the particle travels through the matter until it reaches its stop (at the Bragg peak). The reason for this is that the interaction cross-section increase when the energy of the particle decrease. See Figure 2.7 for a graph showing the deposited energy along the 
path through the matter. This can be exploited in radiotherapy to concentrate the dose to the tumour while keeping the dose to surrounding normal tissue low. However, this also make treatment plans sensitive to range variations.

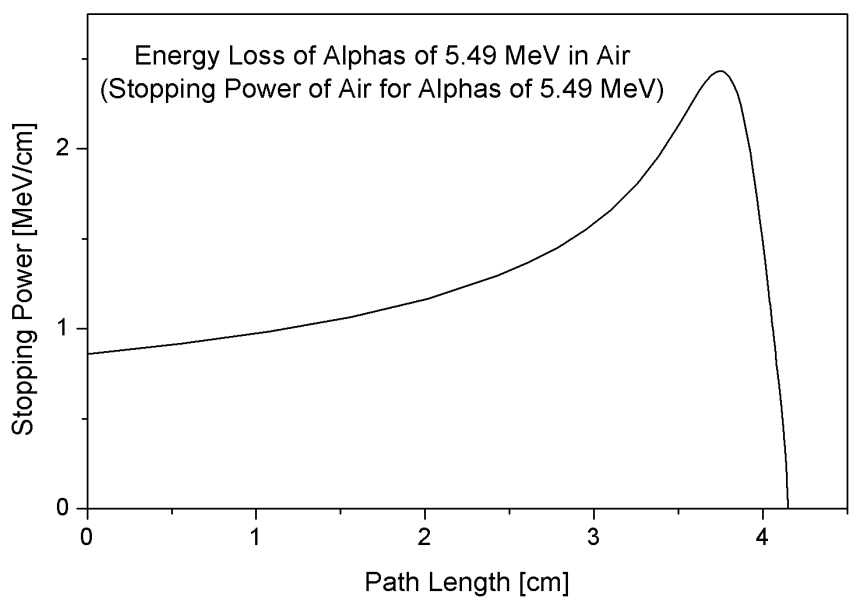

Figure 2.7: A graph showing the energy deposited into air by an alpha-particle of $5.49 \mathrm{MeV}$ along its path.

Another type of external beam radiotherapy is radiosurgery or stereotactic radiation. Based on extremely detailed imaging scans of the, usually small, tumour, radiosurgery targets the tumour with a large number of radiation beams from different angles and directions. The difference between radiosurgery and other types of external beam radiotherapy is not so much how the radiation is delivered but the fundamental concept. Radiosurgery does not utilise that the target and the surrounding normal tissue have different sensitivities to the accumulated dose. Instead it uses a larger number of beam directions which makes it possible to receive a high dose in the tumour without high doses to adjacent normal tissue. Radiosurgery is therefore usually not divided into fractions, and when it is divided into fractions the number of fractions is very small.

\subsubsection{Brachytherapy}

As mentioned above brachytherapy is a form of radiotherapy where the radioactive source is placed inside or next to the area requiring treatment. It is used to treat tumours of for example the cervix, oesophagus, lungs, breasts, skin, and prostate. A key feature of brachytherapy is that the radiation affects only a small area around the source, and since the source is placed directly at the site of the tumour, the healthy tissue further away from the source is exposed to less radiation than with other techniques. Another advantage of brachytherapy is that errors due to patient movement, or movement of the tumour within the body, are reduced since the radioactive sources retain their position in relation to the 
tumour.

Most radioactive sources used for brachytherapy are radionuclides enclosed within a non-radioactive capsule. Different types of radionuclides are used and examples are: Iridium-192 ( $\left.{ }^{192} \mathrm{Ir}\right)$, Iodine-125 $\left({ }^{125} \mathrm{I}\right)$, and Ruthenium-106 $\left({ }^{106} \mathrm{Ru}\right) .{ }^{23}$

The radioactive source is usually inserted using a technique known as afterloading to limit the radiation exposure of the clinical staff. In afterloading, applicators, that are nonradioactive, are positioned in the treatment area and the radioactive source is then subsequently inserted through the applicators. The insertion of the radioactive source could be done by manual afterloading where clinical staff uses appropriate handling tools, or by remote afterloading. When using remote afterloading applicators are after positioning connected to an 'afterloader' machine through connecting guiding tubes. When the clinical staff has left the treatment room the machine applies the source which has until then been inside a radiation shielded safe. ${ }^{23}$

Different types of brachytherapy are classified according to three characteristics:

\section{- Source placement:}

Intracavitary Therapy The applicators and radioactive sources are inserted into an existing body cavity such as the vagina.

Interstitial Therapy The applicators and radioactive sources are inserted directly into tissue using for example needles or wires. This kind of treatment is used for treatment areas such as prostate and breast.

Intralumenal therapy The applicators and radioactive sources are inserted into a lumen, such as the oesophagus.

Intravascular The applicators and radioactive sources are inserted into an artery.

\section{- Duration:}

Temporary The radioactive source is removed after treatment, where the treatment duration is usually between a few minutes and a day.

Permanent Small low dose-rate radioactive seeds are placed into the treatment site and left there to decay. After some time the radiation emitted decays to almost zero, and they can hence remain with no lasting effect.

- Dose-rate No universally accepted definitions exists, however most accept the following:

LDR Low dose-rate corresponds to around 0.5-1 Gy/h.

MDR Medium dose-rate corresponds to around 1-12 Gy/h.

HDR High dose-rate corresponds to above $12 \mathrm{~Gy} / \mathrm{h}$, however typically the doserate is around $2 \mathrm{~Gy} / \mathrm{min}$ which is around 10 times as much.

PDR A specific technique where HDR 'pulses' (typically 5-10 minutes long) are repeated at short intervals (typically once per hour). ${ }^{23}$ 


\subsubsection{HDR brachytherapy for prostate cancer}

Since the focus of our research has been how to optimize dose plans for HDR brachytherapy and especially HDR brachytherapy for prostate cancer, this section will more thoroughly cover this treatment.

The prostate is a male gland located at the top of the urethra, see Figure 2.8. It is a part of the male reproductive system and contributes to the production and storage of seminal fluid. The prostate is normally about three centimeters long and weighs 20 grams for adult males.

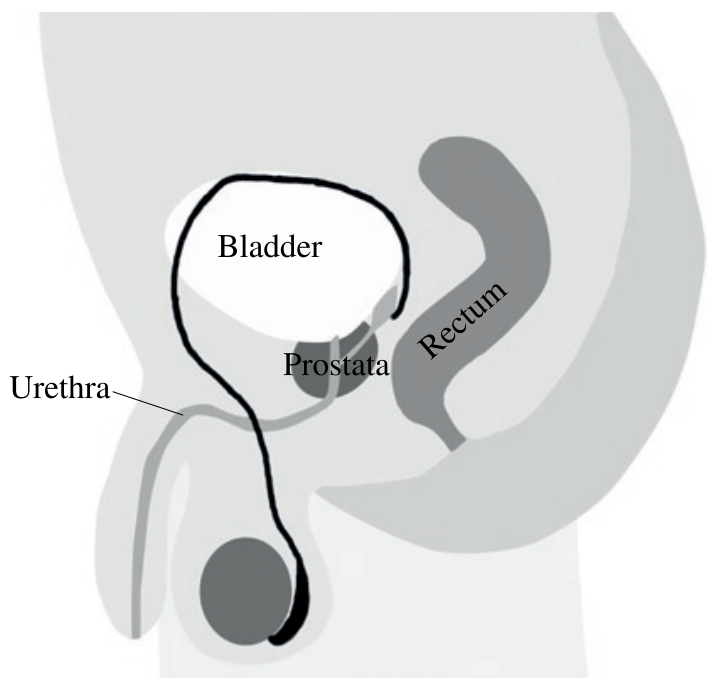

Figure 2.8: Illustration of the prostate and nearby organs.

Prostate cancer is one of the most common types of cancers for men and in 2002 estimations showed that 700000 new cases occurred each year ${ }^{10}$. It mainly affects older men, few are diagnosed before they are fifty, and half are not diagnosed before they are seventy ${ }^{12}$. Even though many persons with prostate cancer never develop symptoms or undergo therapy and the patients eventually die of other causes, $87400^{10}$ deaths were recorded in Europe during 2006.

Possible treatment options for prostate cancer include among other watchful waiting, external beam radiotherapy, high dose-rate (HDR) brachytherapy, low dose-rate (LDR) brachytherapy, and prostatectomy. Which treatment or combination of treatments that is chosen depends on several factors such as the stage of the cancer, age and general health of the patient, patient preferences, and quality of life aspects ${ }^{40}$.

Brachytherapy for prostate cancer was used as a treatment as early as in the 1920's ${ }^{35}$, however the use of remote afterloading with high dose-rate ${ }^{192} \mathrm{Ir}$ was not introduced until the late 1980's ${ }^{39}$. When using HDR brachytherapy for prostate cancer hollow needles, in the following called catheters, are inserted into the treatment volume through the perineum, hence HDR brachytherapy for prostate cancer is an interstitial therapy. Catheters, see Figure $2.9 \mathrm{~b}$ for an example, are usually placed using a fixed template ${ }^{39}$, see Figure 
2.9a for an example of a template, and by using transrectal ultrasound for guidance. For an illustration of HDR brachytherapy for prostate cancer see Figure 2.9c. In Figure 2.9d an equipment setup is shown. The afterloader moves the ${ }^{192} \operatorname{Ir}$ source through the catheters in specified steps, stopping at certain positions called dwell points. The length of a stop is called dwell time. The dwell points are evenly distributed with a distance of $2.5 \mathrm{~mm}$ to $10.0 \mathrm{~mm}$ between possible stopping positions.

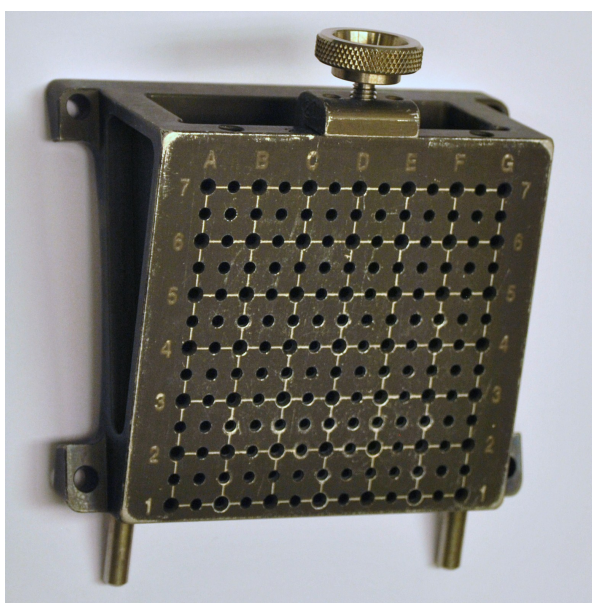

(a) Example of a template for inserting catheters.

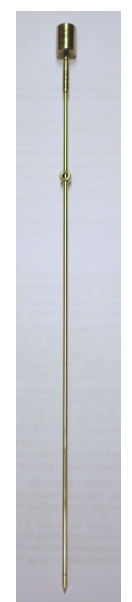

(b) Example of a catheter.

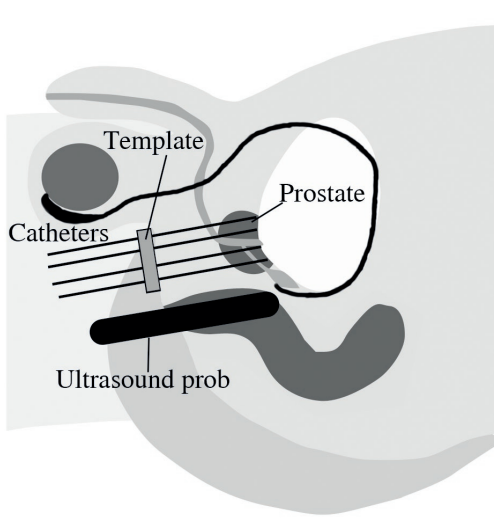

(c) Illustration of the treatment.

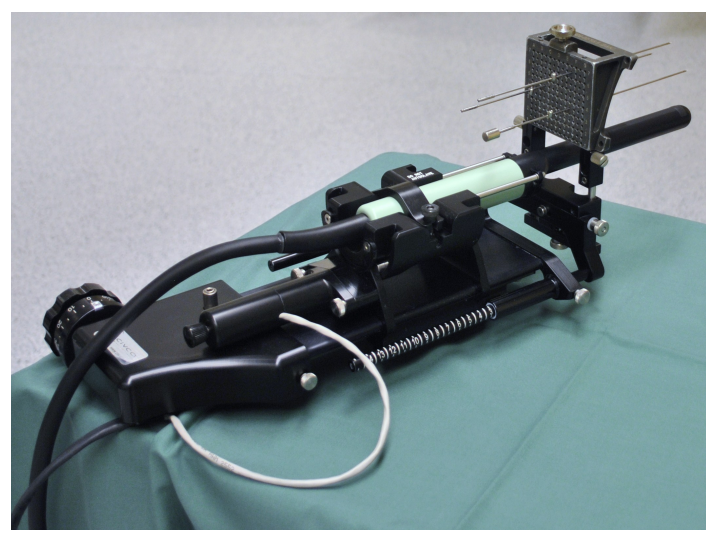

(d) An example of the setup used for the treatment.

Figure 2.9: Equipment used in HDR brachytherapy for prostate cancer.

When constructing treatment plans for HDR brachytherapy the entire prostate is in general considered to be the target. The main organs-at-risk are rectum and urethra, and often also the bladder. 


\subsubsection{Treatment plans}

Before treatment with radiation commence planning is needed, this is often called treatment planning. One of the first steps is to obtain images of the treatment volume using for example CT (computed tomography, which is a kind of X-ray), MRI (magnetic resonance imaging) or ultrasound. This yields a number of cross sections of the treatment volume that together create a 3-D-visualisation (or 3-D-representation) of the treatment volume; in Figure 2.10 an example of such a cross section obtained by ultrasound is found. On these images the target volume and organs-at-risk close to the target volume are contoured. The target volume is usually contoured in different levels:

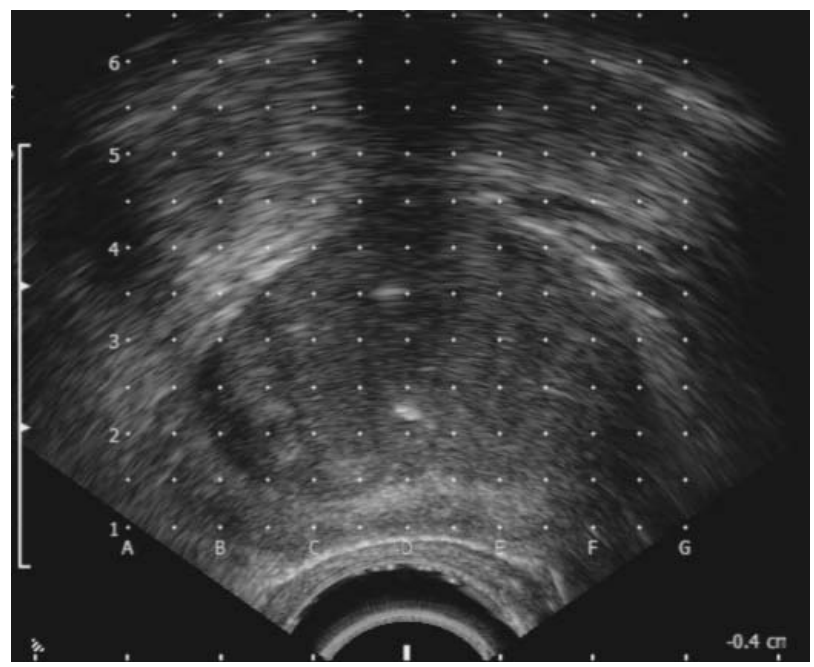

Figure 2.10: Ultrasound image of the treatment area for a prostate cancer patient.

GTV: Gross tumour volume, that is the part of the volume with known tumour growth.

CTV: Clinical Target Volume includes, in addition to volume included in the GTV, also volumes where tumour growth are suspected due to closeness to the tumour or lymph nodes with high probability of spread.

PTV: Planning target volume is the volume that is intended for treatment. It includes the CTV, but also a margin to include possible movement etcetera during treatment.

For each target volume a dose is prescribed, and tolerated dose levels are specified for each organ-at-risk. When defining target volumes and radiation doses the considerations taken are for example:

- The goal of the treatment, that is whether it is intended to be curative (cure the cancer) or palliative (reduce or prevent symptoms caused by cancer).

- Radiosensitivity and growth pattern of the tumour. 
- Risk for side-effects.

- Variations in setup between treatment sessions.

- If and how the treatment is divided into fractions. ${ }^{16}$

Knowing what doses that are desired for each volume, the next step is to decide how to deliver radiation so that the dose conforms as well as possible to the prescription for target volumes and tolerated levels for organs-at-risk. In IMRT this corresponds to choosing directions and sizes for the treatment fields, in permanent LDR brachytherapy it corresponds to choosing positions and strengths of the sources to implant, and for HDR brachytherapy it corresponds to choosing where and for how long to stop the radioactive source. This step is often referred to as creating a dose plan and the goals are:

- To reach the prescribed dose to the target.

- A homogenous dose distribution within the target.

- As low dose as possible (and below the tolerated dose level) to organs-at-risk.

- As low total dose as possible.

- A plan that is realisable and can be repeated with high precision.

It is generally impossible to fulfil all the goals, so trade-offs are inevitable. ${ }^{16}$

In most cases the dose plan is created for the entire treatment volume simultaneously; this is called 3-D planning. There are different ways to generate the dose plan. Some generate dose plans manually by iteratively changing how the radiation is delivered and evaluating the generated dose distribution (the dose to each point). Others use software that generates plans by using different kinds of optimization techniques, in Section 3.3 some of these optimization techniques are presented.

There are other steps included in treatment planning as well, such as plans for fixation and dose simulations; however these will not be covered since they do not affect the optimization process.

\subsubsection{Evaluation of dose plans}

Before applying a dose plan to a patient, evaluation is needed. One common method for evaluation is to graphically illustrate the dose distribution and visually inspect it. Visual evaluation provides information about if and where hot spots (volumes receiving a very high dose) and cold spots (volumes receiving a low dose) are located and the size of such volumes. It is also quite easy to see how the dose conforms to the target. In Figure 2.11 an example of how the dose can be illustrated to enable visual inspection is found.

\section{Dose-volume histograms}

Another common method for evaluation of dose plans is dose-volume histograms (DVH) which describe the dose distribution for a structure. There are a few different types of DVH:s, but the dominating two are the cumulative DVH and the differential DVH. A differential DVH illustrates for each possible dose how large part of the volume that receives 


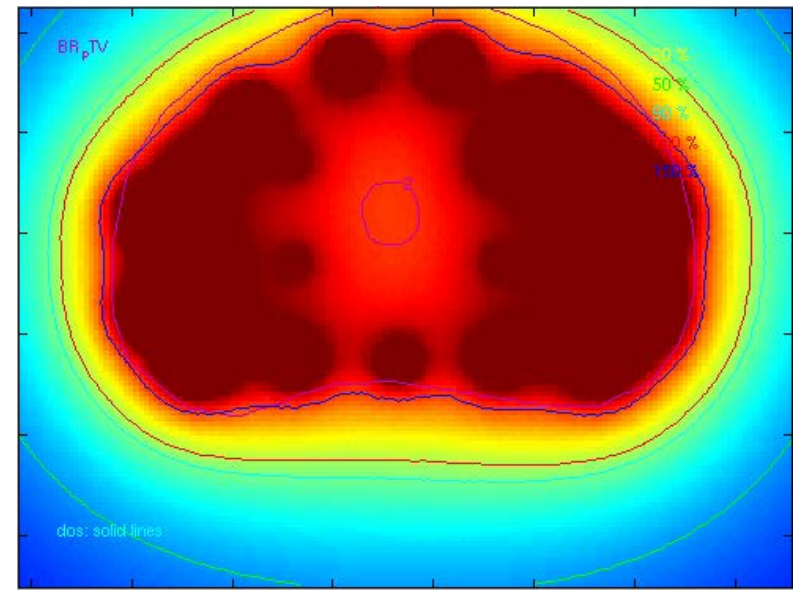

Figure 2.11: Graphical illustration of the dose distribution in a cross section. Red colours represent high doses and blue colours represent low doses.

exactly that dose, see Figure 2.12b for an illustration. A cumulative DVH on the other hand illustrates how much of the volume that receives a certain dose or more (for each possible dose), see Figure 2.12a for an example. Cumulative DVH:s are more common and hence when we write only DVH we refer to the cumulative DVH. Ideally the entire target volume receives exactly the prescription dose, corresponding to the DVH in Figure 2.13a, while organs-at-risk receive no dose at all, corresponding to the DVH in Figure $2.13 b$.

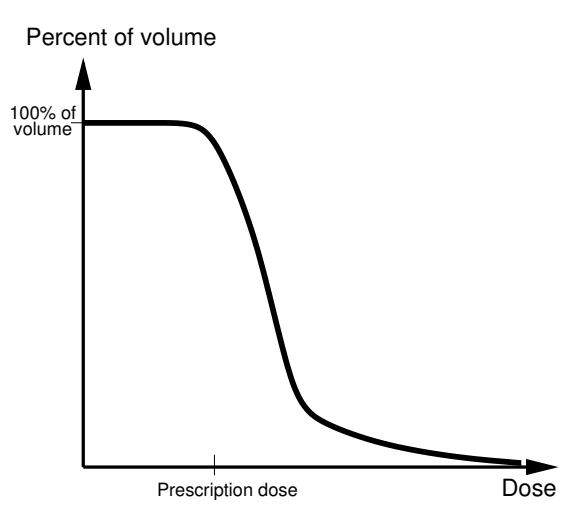

(a) Cumulative DVH.

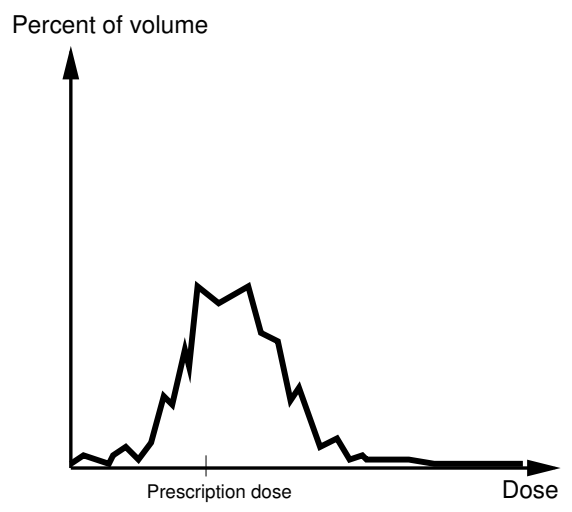

(b) Differential DVH.

Figure 2.12: The two types of dose-volume histograms (DVH). 


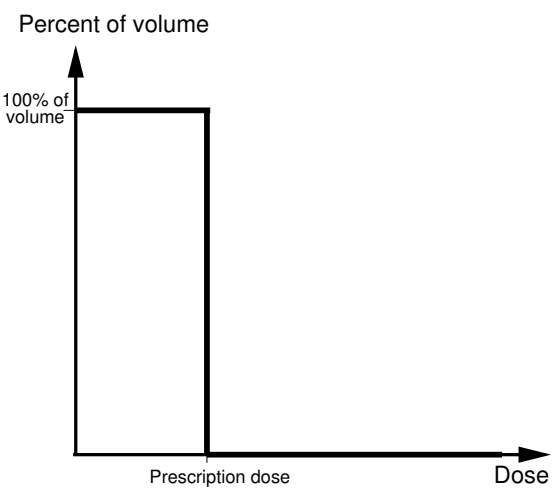

(a) Ideal cumulative $\mathrm{DVH}$ for target.

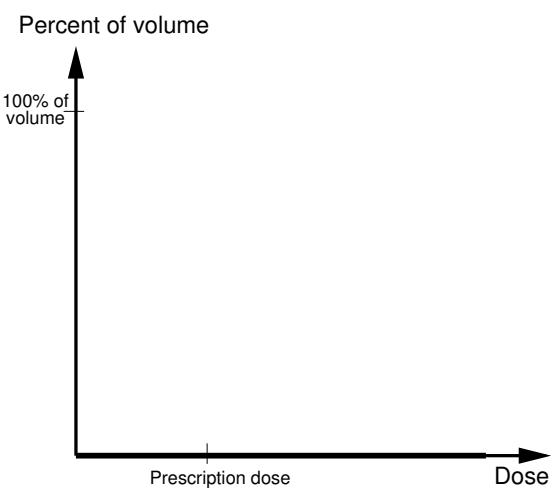

(b) Ideal cumulative DVH for organ-atrisk.

Figure 2.13: Ideal dose-volume histograms.

\section{Quantitative evaluation measures}

When statistically analysing different plans, and also when prescribing a plan for a patient, it is convenient to express the quality of the plan as one or a few numbers. Many measures of this type have been suggested in literature and here we describe some of these.

The two most common types of quantitative measures are extracted from the DVH, and these types of measures are often called dose-volume parameters, dosimetric indices, or DVH-based parameters. One type of dosimetric index is $V_{x}^{y}$, which corresponds to the percentage of volume $y$ that receives $x$ percent of the prescription dose ( $x$ is sometimes expressed in Gy instead of a percentage of the prescription dose). As an example of this $V_{100}^{\mathrm{CTV}}$ measures the percentage of the CTV that receives the prescription dose or more. The other type of dosimetric index is $D_{x}^{y}$, which is the reversal of $V_{x}^{y}$, and hence correspond to the lowest dose received by the highest receiving $x$ percent of structure $y$ 's volume ( $x$ is sometimes expressed in cc instead of a percentage of the total volume of the structure). As an example of this $D_{90}^{\text {CTV }}$ measures the lowest dose received by $90 \%$ of the CTV. Figure 2.14 shows how $V_{x}^{y}$ and $D_{x}^{y}$ can be extracted from the DVH.

Another aspect of a treatment plan that is quantified is the homogeneity. Several different measures have been proposed, such as the dose non-uniformity ratio ${ }^{49}$, the conformation number ${ }^{54}$, the dose volume uniformity index ${ }^{41}$ etcetera. The dominating measure used to quantify homogeneity is however the homogeneity index (HI). It was introduced by $\mathrm{Wu}$ et al. ${ }^{55}$ and is defined as the fraction of the target volume that recieves a dose between $100 \%$ and $150 \%$ of the prescription dose, and can be expressed in the following way,

$$
H I=1-\frac{V_{150}^{\mathrm{PTV}}}{V_{100}^{\mathrm{PTV}}} .
$$

One measure that has been used to some extent is the conformal index (COIN), which was presented by Baltas et al. ${ }^{5}$. COIN quaintifies how well the prescription dose covers 


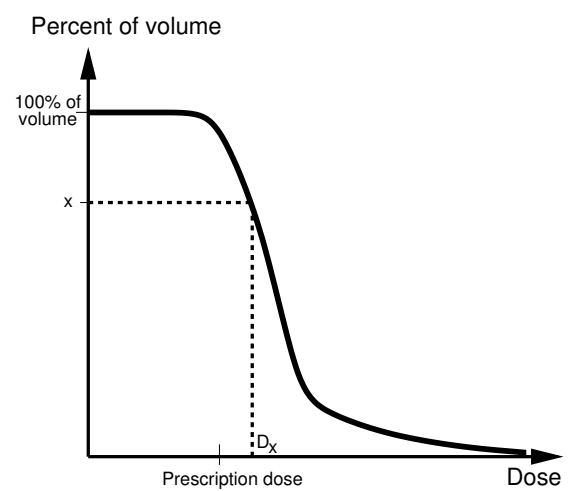

(a) Illustration of how $D_{x}$ is calculated.

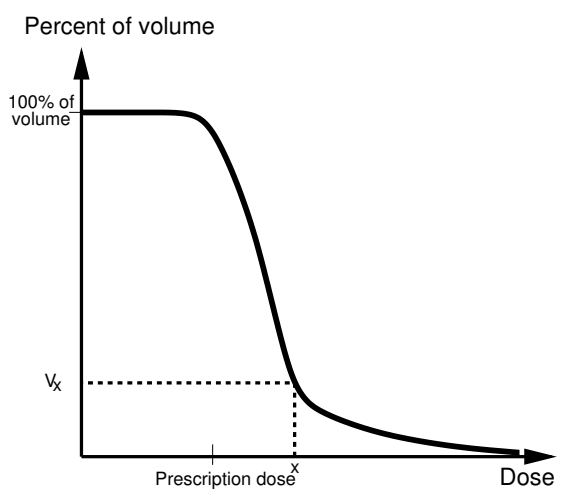

(b) Illustration of how $V_{x}$ is calculated.

Figure 2.14: Illustration of how dosimetric indices can be obtained from the dosevolume histograms.

the target, how much normal tissue that is covered by the prescription dose, and unwanted irradiation of critical structures. It can take values between 0 and 1 , where 1 corresponds to the ideal situation.

The last measure we will cover here is the generalized equivalent uniform dose (gEUD). Niemierko ${ }^{37}$ introduced a concept called equivalent uniform dose (EUD) that can be used to compare inhomogeneous dose distributions. It is related to the radiobiological effect, and for any dose distribution EUD is the dose (in Gy), which when distributed uniformly across the target volume causes the same radiobiological effect. Niemierko ${ }^{38}$ later introduced gEUD which is an estimation of EUD. One advantageous property of gEUD is that it is either a convex or a concave function of the dose. To find the value of gEUD for a volume the first step is to discretize the volume into a collection of points $N$. Then

$$
\operatorname{gEUD}_{a}=\frac{1}{|N|} \sum_{n \in N} \operatorname{Dose}_{n}^{a}
$$

where $a$ is a parameter, and Dose $_{n}$ is the dose in point $n$.

\subsubsection{Dosimetric protocols}

In this section we will describe some dosimetric protocols used for HDR brachytherapy for prostate cancer. A dosimetric protocol describes the constraints and/or goals that the planner tries to satisfy when creating a plan for the patient. The American Brachytherapy Society (ABS) has published recommendations ${ }^{57}$ for how HDR brachytherapy should be used for prostate cancer. In these guidelines they state that the prescription dose should cover at least $90 \%$ of the target volume $\left(V_{100}^{\mathrm{CTV}}>90 \%\right)$, and that $V_{100}^{\mathrm{CTV}}>95 \%$ should be the expactation. They do however not present any guidelines regarding OAR or overdosage of the target. The reason for this is that the literature presents several different 
dosimetric protocols, which all correspond to similar excellent outcomes. The European counterpart of ABS GEC/ESTRO-EAU has also published recommendations ${ }^{29,24}$ for HDR brachytherapy. Just like ABS they do not provide a dosimetric protocol for the same reason, only a few bounds for the urethra, penile bulb and rectum. In Table 2.1 we present a number of examples of dosimetric protocols that have been used clinically.

Table 2.1: Examples of dosimetric protocols. Unknown for the target constraints mean that the reference we have does not include constraints for the target.

\begin{tabular}{|c|c|c|}
\hline Institution & Target constraints & OAR constraints \\
\hline $\begin{array}{l}\text { University of Califor- } \\
\text { nia San Francisco } \\
57\end{array}$ & unknown & $\begin{array}{l}V_{75}^{\text {Bladder }}<1 \mathrm{cc}, V_{125}^{\text {Urethtra }}<1 \mathrm{cc}, \\
V_{150}^{\text {Urethtra }}=0 \mathrm{cc}, V_{75}^{\text {Rectum }}<1 \mathrm{cc}\end{array}$ \\
\hline $\begin{array}{l}\text { University of } \\
\text { Toronto }^{57}\end{array}$ & unknown & $\begin{array}{l}D_{10}^{\text {Urethtra }}<118 \%, D_{0}^{\text {Urethtra }}<125 \% \\
V_{80}^{\text {Rectum }}<0.5 \mathrm{cc}\end{array}$ \\
\hline $\begin{array}{l}\text { Radiation therapy } \\
\text { oncology group }\end{array}$ & $V_{100}^{\mathrm{CTV}}>90 \%$ & $\begin{array}{l}V_{75}^{\text {Bladder }}<1 \mathrm{cc}, V_{75}^{\text {Rectum }}<1 \mathrm{cc}, \\
V_{125}^{\text {Urethtra }}<1 \mathrm{cc}\end{array}$ \\
\hline $\begin{array}{l}\text { Klinikum Offenbach } \\
\text { GmbH }^{6}\end{array}$ & $\begin{array}{l}V_{100}^{\mathrm{CTV}} \geq 90 \% \\
D_{90}^{\mathrm{CTV}} \geq 100 \% \\
V_{150}^{\mathrm{CTV}} \leq 35 \% \\
\end{array}$ & $\begin{array}{l}D_{10}^{\text {Urethra }} \leq 115 \%, D_{0.1 c c}^{\text {Urethra }} \leq 120 \% \\
D_{10}^{\text {Rectum }} \leq 75 \%, D_{0.1 c c}^{\text {Rectum }} \leq 80 \% \\
D_{10}^{\text {Bladder }} \leq 75 \%, D_{0.1 c c}^{\text {Bladder }} \leq 80 \%\end{array}$ \\
\hline
\end{tabular}





\section{Optimization of Radiotherapy}

This chapter will introduce the reader to optimization of dose plans in radiotherapy, starting with a general problem formulation and then presenting the framework needed for performing the optimization. The chapter ends with presenting earlier research related to our research.

\subsection{Problem formulation}

Optimization within the field of radiotherapy is mostly focused on creating good ('nearoptimal') treatment plans. A quite general problem formulation is:

Given the patient's anatomy, what is the best way to deliver a tumoricidal (lethal) dose to the cancerous region, while simultaneously limiting the dose of radiation to organs-at-risk surrounding the cancer so that they can survive the treatment.

What needs to be determined is thus how to deliver the dose, that is, creating a dose plan.

Translating the general problem formulation into an optimization model is difficult. One reason is that the goal of a tumoricidal dose of radiation to the cancerous region conflicts with limiting the dose to organs-at-risk. Another difficulty is that if a region receives an unreasonably high dose, then all cells within this region die, and if the region is large enough this will cause an unwanted condition of tissue death called necrosis. Yet another difficulty is that different organs react to radiation in different ways. ${ }^{22}$

The treatment goal may also vary between patients; in many cases it is of course to deliver a tumoricidal dose of radiation to the cancerous region while keeping the dose to organs-at-risk under control. However, for terminally ill patients the goal is not to cure the cancer, but rather to increase quality of life. This might for example mean that minimizing the dose to certain organs-at-risk is more important than a tumoricidal dose, for such patients. There are also cases when the likelihood of success is very low when sparing 
organs-at-risk. Then the question of to what degree the patient is willing to risk damage to the organs-at-risk arises. Hence there are many alternative goals and constraints that the optimization models should be able to accommodate, and flexibility is therefore important. $^{22}$

A part of creating an optimization model is to translate goals and constraints into mathematical functions. Since both goals and constraints usually are qualitative, such as spare an organ-at-risk, it is often hard to find suitable functions. This, together with the difficulties above and variations in goal, have resulted in the development of many different models, and in Section 3.3 some of these are presented. All these models do however try to solve the same problem, namely the general problem formulation above.

\subsection{Framework}

To construct an optimization model for the general problem formulation it is necessary to describe how the dose distribution depends on the chosen variables. A natural question that arises in this context is where the dose should be calculated. It is clear that it is not possible to calculate it for every single cell, so some kind of discretization is needed. In Section 3.2.1 different choices of discretizations are described, all yielding a number of points that represent the total treatment volume. The generated points are called dosecalculation points. For each of these points a description of how the dose depends on the variables is needed, and in Section 3.2.2 this description is given for the case of HDR brachytherapy.

\subsubsection{Dose point generation}

There are a number of different suggestions for how to discretize the treatment volume and the choice depends on for example what the points should be used for, and the kind of treatment considered. When evaluating dose plans it is common to use a very fine square grid, where each point in the grid represents a small part of the treatment volume. We have when evaluating our generated dose plans used such a square grid where each point represents $0.25 \mathrm{~mm}^{3}$ of the treatment volume. To use such a fine grid during the optimization phase would lead to very large problem instances. With a resolution of 0.25 $\mathrm{mm}^{3}$ per point, it requires around 2 million points to cover the treatment volume for prostate HDR brachytherapy. For this reason it is common to use a sparser resolution for the optimization.

When considering IMRT it is common to use a square grid also for the optimization phase, but letting each point represent a larger volume. In IMRT this works well since the dose distributions are quite uniform and hence a high level of accuracy can be achieved using quite few points. Dose distributions in brachytherapy on the other hand are significantly more nonuniform and more care is needed when choosing points. The common technique to generate dose-calculation points in brachytherapy seems to be the one presented by Lahanas et al. ${ }^{30}$ and this is the technique we have used. Here the dose-calculation points are generated both within the volume of each structure and on the surface of the volume of each structure. The points within each structure are generated by using triplets of Sobol sequences (a type of quasi-random low-discrepancy sequences) 
inside a bounding box of the structure, and each point is then investigated to determine if it is inside the structure or not. To generate surface points requires a triangulation of the surface of the structure. Given the triangulation, the number of points to generate in each triangle is chosen using the Stochastic Universal Sampling Algorithm. Then for each triangle the chosen number of points are randomly distributed by the random generation of barycentric coordinates. ${ }^{30}$

\subsubsection{Dose calculations}

When calculating doses the first step is usually to calculate the dose-rate contribution from one dwell point (seed or beamlet) to one dose-calculation point per time unit. Given these dose-rates it is easy to calculate the total dose in a dose-calculation point by multiplying the dose-rate with the dwell time for each dwell point and then summing this up. This means that if $d_{i j}$ is the dose-rate contribution from dwell point $j$ to dose-calculation point $i$, then the total dose in dose-calculation point $i$ amounts to $\sum_{j} d_{i j} t_{j}$, where $t_{j}$ is the dwell time in position $j$.

As mentioned in Section 2.5.4 the usual radioactive source in HDR prostate brachytherapy is ${ }^{192}$ Ir. Iridium-192 decays by beta and photon emission ${ }^{23}$. All ${ }^{192}$ Ir sources for HDR brachytherapy are very similar, regardless of who the manufacturer is. They consist of a cylinder of ${ }^{192} \mathrm{Ir}$ enclosed in stainless steel. The steel enclosure is thick enough to stop all electrons emitted by $\beta$-decay. However, the photons with high energy are only a little affected by the steel and can hence be used for the treatment.

Below we describe how the dose-rate contributions can be calculated by functions fitted to the dose distribution in water around a single HDR ${ }^{192} \mathrm{Ir}$ source (Nucletron microSelectron HDR v1). This method was presented by Russell et al. ${ }^{48}$ and the dose-rate contributions obtained by this method are equivalent to those obtained using the AAPM TG43 formalism ${ }^{42}$.

By Monte Carlo simulations of photons in a geometry consisting of the source placed in a large volume of water one obtains a good approximation of the dose-rate from an ${ }^{192} \mathrm{Ir}$ source to the prostate. The results obtained by these simulations can be parameterised in different ways; one of these is described below.

The dose-rate at a point, $D R_{t o t}$, as a function of the distance to the center of the source (in polar coordinates $r$ and $v$, see Figure 3.1) is calculated as:

$$
\begin{gathered}
{\left[D R_{\text {tot }}(r, v)\right]_{M C}=\left[D R_{\text {prim }}(r, v)\right]_{M C}+\left[D R_{\text {scat }}(r, v)\right]_{M C}} \\
{\left[D R_{\text {prim }}(r, v)\right]_{M C}=\frac{1}{4 \pi r^{2}} a_{1}(v) e^{-\left(a_{2}(v)-a_{3}(v) r\right) r}} \\
{\left[D R_{\text {scat }}(r, v)\right]_{M C}=\frac{1}{4 \pi r^{2}}\left(b_{1}(v)\left[\left(1+b_{2}(v)\right)-e^{-b_{3}(v) r}\right] e^{-b_{4}(v) r}\right) .}
\end{gathered}
$$

Here, $a_{1}, a_{2}, a_{3}, b_{1}, b_{2}, b_{3}$, and $b_{4}$ are parameters that depend on $v$. Note that this is not the actual dose-rate obtained at the hospital, thereof the index $M C$. At the hospital one has to adjust the dose-rate to the strength of the source that is actually used. The reason for this is that the strength depends on how old the source is, since the activity 


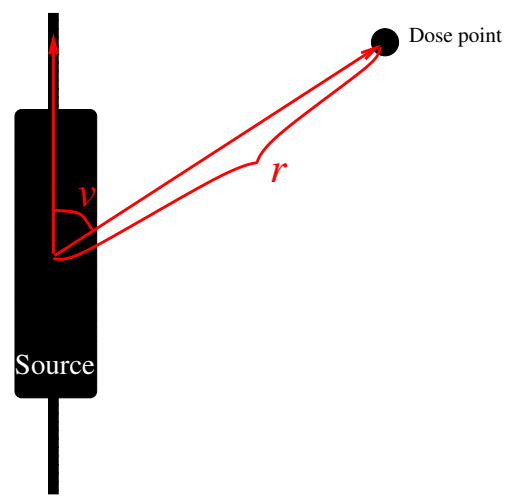

Figure 3.1: Illustration of how to measure the angle $(v)$ and radius $(r)$ for calculating doses when using an ${ }^{192}$ Ir source in HDR brachytherapy of the prostate.

decreases due to the radioactive decay. The strength of the source is measured in the physical quantity reference air kerma rate (RAKR), and the actual dose-rate is

$$
\left[D R_{\text {tot }}(r, \theta)\right]_{\text {hospital }}=\frac{[R A K R]_{\text {hospital }}}{[R A K R]_{M C}}\left[D R_{\text {tot }}(r, \theta)\right]_{M C},
$$

where $[R A K R]_{M C}$ is a known constant.

\subsection{Earlier models and research}

The first model for optimizing dose plans for radiotherapy was proposed already in 1968 by Bahr et al. ${ }^{4}$ and it was linear. Since then many different models and methods have been proposed. Good comprehensive reviews of external radiotherapy optimization literature have been presented by Shepard et al. ${ }^{50}$ and Ehrgott et al. ${ }^{17}$. De Boeck et al. ${ }^{14}$ present a good review of HDR brachytherapy where they also classify different approaches according to criteria such as optimization technique and objective type. Below we focus on models and methods for HDR brachytherapy. Some results from external radiotherapy of importance for our research are also presented, but the modality can be assumed to be HDR brachytherapy if not explicitly stated otherwise.

\subsubsection{Subproblems}

A dose plan usually contain several decisions that could be optimized, regardless of the type of radiotherapy. For HDR brachytherapy there is mainly two decisions:

B1. Catheter positioning: Deciding where the catheters should be placed. It might also include choosing the number of catheters to place. Catheter positioning is also called catheter placement. 
B2. Dwell time distribution: Choosing how long the source dwell in each possible dwell point. Dwell time distribution is also called dwelling time pattern.

For IMRT there is three decisions:

I1. The beam angle optimization problem: Selecting the number of beams and the directions from which the gantry delivers radiation. Also called the geometry problem.

I2. The fluence map optimization problem: Choosing the intensity pattern that should be used for each beam (that is the intensity of each beamlet). Also referred to as the intensity problem.

I3. The segmentation problem: Deciding how to operate the multileaf collimator to efficiently administer the treatment. Also called the realization problem.

The segmentation problem does not have a correspondence in HDR brachytherapy. The catheter positioning problem and the beam angle optimization problem are similar, and the general approach for both seems to be to generate a number of possible positions (angles) and choose the subset that yield the best dwell time distribution (fluence map).

Fluence map optimization is very similar to dwell time distribution, and hence such models are very similar. There are however fundamental differences in which dose distributions that are possible to achieve by changes in intensity (time), and this requires special considerations. In HDR brachytherapy dose peaks are inevitable, in IMRT on the other hand such peaks do not, or should at least not, occur. An example of how this difference affects models is that hard dose constraints (constraints limiting the dose in a dose-calculation point to a certain value) are less applicable in brachytherapy.

\subsubsection{Penalty models for dwell time distribution optimization}

Almost all optimization methods and models for HDR brachytherapy have been presented during the last fifteen years. The majority of these seem to utilise a common concept; it is assumed that physicians provide upper and lower limits on the dose for each structure in the treatment volume (tumours, organs-at-risk and other healthy tissue) and the models then penalise values above or below these limits. The objective function of all such models is to minimize the penalty. The difference between models is how the deviation is penalised, and if the penalty for each structure is treated as a separate objective (multi-objective problems) or if a weighted sum of the penalties is used (single-objective problem). A general model of this type, formulated in a way that makes it easy to understand, but not necessarily to solve, is presented in Table 3.1.

In HDR brachytherapy several authors have presented penalty models that can be represented by the general model, for example Milickovic et al. ${ }^{34}$, Lahanas et al. ${ }^{31}$ and Lessard and Pouliot ${ }^{32}$. The most prevalent formulation in IMRT is also a penalty model, where $H(x)$ is the Heaviside function and $p=2 .{ }^{17}$ Jozsef et al. ${ }^{27}$ also use a penalty model, but their model can not be represented by the general model. The reason is that unlike the general model that considers the deviation at each point separately they only consider the maximum deviation. 


\section{Parameters and sets}

$S \quad=$ Set of structures.

$N_{s} \quad=$ Set of dose-calculation points in structure $s$.

$T=$ Set of dwell points.

$D_{\text {min }}^{s}=$ Lower dose limit for structure $s$.

$D_{\max }^{s}=$ Upper dose limit for structure $s$.

$d_{i j}^{s} \quad=$ Dose-rate contribution from dwell point $j$ to dose-calculation point $i$ in structure $s$.

$\lambda_{\min }^{s}=$ Objective weight for underdosage of structure $s$.

$\lambda_{\max }^{s}=$ Objective weight for overdosage of structure $s$.

$p \quad=$ Model dependent parameter.

$H(x)=$ Heaviside function or an approximation of the Heaviside function.

\section{Variables}

$\operatorname{Dose}_{i}^{s}=$ Dose to dose-calculation point $i$ in structure $s$.

$\underline{\Delta}_{i}^{s} \quad=$ Underdosage of dose-calculation point $i$ in structure $s$.

$\bar{\Delta}_{i}^{s} \quad=$ Overdosage of dose-calculation point $i$ in structure $s$.

$t_{j} \quad=$ Dwell time at point $j$.

Objective function in single objective case

$$
\min \sum_{s \in S} \sum_{i \in N_{s}}\left(\lambda_{\min }^{s} H\left(\underline{\Delta}_{i}^{s}\right)\left(\underline{\Delta}_{i}^{s}\right)^{p}+\lambda_{\max }^{s} H\left(\bar{\Delta}_{i}^{s}\right)\left(\bar{\Delta}_{i}^{s}\right)^{p}\right)
$$

Objective function in multi-objective case

$$
\begin{gathered}
\min f(\Delta)=\left(f_{\text {min }}^{s}(\underline{\Delta}) \quad \forall s \in S, \quad f_{\text {max }}^{s}(\bar{\Delta}) \quad \forall s \in S\right) \\
f_{\text {min }}^{s}(\underline{\underline{\Delta}})=\sum_{i \in N_{s}} H\left(\underline{\Delta}_{i}^{s}\right)\left(\underline{\Delta}_{i}^{s}\right)^{p} \quad \forall s \in S \\
f_{\text {max }}^{s}(\bar{\Delta})=\sum_{i \in N_{s}} H\left(\bar{\Delta}_{i}^{s}\right)\left(\bar{\Delta}_{i}^{s}\right)^{p} \quad \forall s \in S
\end{gathered}
$$

Subject to

$$
\begin{aligned}
\operatorname{Dose}_{i}^{s} & =\sum_{j \in T} d_{i j}^{s} t_{j} & & \forall s \in S, i \in N_{s} \\
\Delta_{i}^{s} & =D_{\text {min }}^{s}-\operatorname{Dose}_{i}^{s} & & \forall s \in S, i \in N_{s} \\
\bar{\Delta}_{i}^{s} & =\operatorname{Dose}_{i}^{s}-D_{\text {max }}^{s} & & \forall s \in S, i \in N_{s} \\
t_{j} & \geq 0 & & \forall j \in T
\end{aligned}
$$

Table 3.1: A general description of many of the proposed models for HDR brachytherapy. 
The model that seems to be most used in clinical practice is the general model with $p=1$ and where $H(x)$ is the Heaviside function. It was first introduced by Lessard and Pouliot ${ }^{32}$, who solve it using fast-simulated annealing. They refer to their method as IPSA (inverse planning simulated annealing). In 2006, Alterovitz et al. ${ }^{2}$ showed that this model can be formulated as a linear problem, and hence can be solved easily to global optimality by using for example the simplex method. This linear model, which we in the following will refer to as the linear penalty model, is presented in Table 3.2.

For plans generated by solving the linear penalty model it is common with a few dominating positions with dwell times that are large compared to the others ${ }^{6,13}$. However, long dwell times cause hot spots around the corresponding positions. Concern has been expressed regarding the unknown effect of such hot spots and therefore more homogeneous solutions are preferred ${ }^{13}$. A few attempts to deal with the problem of long dwell times have been introduced. Chanjon et al. ${ }^{13}$ suggest that artificial normal tissue is introduced around the catheters or that explicit ceilings on maximum dwell time are added. Baltas et al. ${ }^{6}$ introduce dwell time gradients, that limit the dwell time difference between adjacent points, and their results using these are promising ${ }^{33}$.

\subsubsection{Dwell time distribution optimization models that include evaluation measures}

During the past few years there has been an increasing interest in models that more directly correspond to the measures used clinically to evaluate dose plans. As mentioned in Section 2.5.6 two of the most common quantitative evaluation measures are the dosimetric indicies $D_{x}^{y}$ and $V_{x}^{y}$. The research groups Beliën et al. ${ }^{7}$, Siauw et al. ${ }^{51}$ and Gorissen et al. ${ }^{21}$ have all presented models that can optimize $V_{x}^{y}$, while constraining $D_{x}^{y}$ and $V_{x}^{y}$. Their models are all very similar, and the main component used to include these measures is binary variables for each pair of dose-calculation point and dosimetric index. A general model of this type is presented in Table 3.3.

Both Beliën et al. and Siauw et al. conclude that it is not possible to find an optimal solution within any reasonable time frame when using standard mixed integer programming software to solve the model, and they therefore construct heuristics. The heuristic by Siauw et al. start by relaxing the binary restriction (Equation 3.3e) and the constraints on dosimetric indices (Equation 3.3c), and then solve the resulting linear programming problem. Thereafter constraints of the type

$$
\operatorname{Dose}_{i}^{s} \leq D_{k}^{s}
$$

are added to the $\left|N_{s}\right|\left(1-U_{s}\right)$ dose-calculation points in $N_{s}$ that received the least dose in the optimal solution of the linear programming problem. The problem is then solved again, which yields a solution that satisfy all upper constraints on dosimetric indices, although there is no guarantee that the lower bounds are satisfied.

Beliën et al. present an approach which alternates between solving linear programming problems and a simulated annealing neighbourhood search. The search is guided by both primal and dual information obtained when solving the linear programming problem.

Gorissen et al. ${ }^{21}$ manage to solve their model to optimality within reasonable computing time. Their model is however equivalent to that by Siauw et al., who say they can not solve an instance of reasonable size to optimality given several hours. The low computing 


\section{Parameters}

$S \quad=$ Set of structures.

$N_{s} \quad=$ Set of dose-calculation points in structure $s$.

$T=$ Set of dwell points.

$D_{\min }^{s}=$ Lower dose limit for structure $s$.

$D_{\text {max }}^{s}=$ Upper dose limit for structure $s$.

$M_{\text {min }}^{s}=$ Penalty for underdosage of structure $s$.

$M_{\max }^{s}=$ Penalty for overdosage of structure $s$.

$d_{i j}^{s} \quad=$ Dose-rate contribution from dwell point $j$ to dose-calculation point $i$ in structure $s$.

\section{Variables}

$\operatorname{Dose}_{i}^{s}=$ Dose to dose-calculation point $i$ in structure $s$.

$w_{i}^{s} \quad=$ Penalty for dose-calculation point $i$ in structure $s$.

$t_{j} \quad=$ Dwell time at point $j$.

Objective function

$$
\min \sum_{s \in S} \sum_{i \in N_{s}} \frac{w_{i}^{s}}{\left|N_{s}\right|}
$$

Subject to

$$
\begin{array}{rlrl}
w_{i}^{s}+M_{s}^{\min } \sum_{j \in T} d_{i j}^{s} t_{j} \geq M_{s}^{\text {min }} D_{s}^{\text {min }} & & \forall s \in S, i \in N_{s} \\
w_{i}^{s}-M_{s}^{\max } \sum_{j \in T} d_{i j}^{s} t_{j} \geq-M_{s}^{\text {max }} D_{s}^{\max } & & \forall s \in S, i \in N_{s} \\
w_{i}^{s} \geq 0 & & \forall s \in S, i \in N_{s} \\
t_{j} \geq 0 & \forall j \in T
\end{array}
$$

\section{Alternative equivalent formulation}

Objective function

$$
\min \sum_{s \in S} \sum_{i \in N_{s}} \frac{M_{s}^{\text {min }}}{\left|N^{s}\right|}\left(D_{s}^{\text {min }}-D_{o s} e_{i}^{s}\right)^{+}+\sum_{s \in S} \sum_{i \in N_{s}} \frac{M_{s}^{\max }}{\left|N^{s}\right|}\left(\text { Dose }_{i}^{s}-D_{s}^{\text {max }}\right)^{+}
$$

Subject to

$$
\begin{aligned}
\operatorname{Dose}_{i}^{s} & =\sum_{j \in T} d_{i j}^{s} t_{j} & & \forall s \in S, i \in N_{s} \\
t_{j} & \geq 0 & & \forall j \in T
\end{aligned}
$$

Table 3.2: The linear penalty model which is the model usually used in clinical practice when optimizing dose plans. It was originally presented by Alterovitz et al. ${ }^{2}$. Here $(x)^{+}=\max (0, x)$ 


\section{Parameters}

$S \quad=$ Set of structures.

$N_{s} \quad=$ Set of dose-calculation points in structure $s$.

$T=$ Set of dwell points.

$V_{s} \quad=$ Set of dosimetric indices for structure $s$.

$D_{k}^{s} \quad=$ Threshold dose for dosimetric index $k$ for structure $s$.

$L_{k}^{s} \quad=$ Lower bound on dosimetric index $k$ for structure $s$.

$U_{k}^{s} \quad=$ Upper bound on dosimetric index $k$ for structure $s$.

$M \quad=$ A sufficiently large number.

$d_{i j}^{s} \quad=$ Dose-rate contribution from dwell point $j$ to dose-calculation point $i$ in structure $s$.

\section{Variables}

$\operatorname{Dose}_{i}^{s}=$ Dose to dose-calculation point $i$ in structure $s$.

$y_{i k}^{s}= \begin{cases}1 & \text { if } D_{o s e}^{s} \geq D_{k}^{s} \\ 0 & \text { otherwise }\end{cases}$

$t_{j} \quad=$ Dwell time at point $j$.

Objective function

$$
\max \sum_{i \in N_{s_{o}}} y_{i k_{o}}^{s_{o}}
$$

Subject to

$$
\begin{aligned}
\text { Dose }_{i}^{s} & =\sum_{j \in T} d_{i j}^{s} t_{j} & & \forall s \in S, i \in N_{s} \\
D_{k}^{s} y_{i k}^{s} & \leq \operatorname{Dose}_{i}^{s} \leq D_{k}^{s}+M y_{i k}^{s} & & \forall s \in S, i \in N_{s}, k \in V_{s} \\
\left|N_{s}\right| L_{s} & \leq \sum_{i \in N_{s}} y_{i k}^{s} \leq\left|N_{s}\right| U_{s} & & \forall s \in S, k \in V_{s} \\
t_{j} & \geq 0 & & \forall j \in T \\
y_{i k}^{s} & \in\{0,1\} & & \forall s \in S, i \in N_{s}, k \in V_{s}
\end{aligned}
$$

Table 3.3: A general model for optimization of $D_{x}^{y}$ and $V_{x}^{y}$. 
times obtained by Gorissen are therefore probably a result of them including only a very small number of constraints.

Also Ruotsalainen et al. ${ }^{47}$ have presented an optimization approach that include $V_{x}^{y}$. Their approach is an interactive multi-objective approach, and they use several types of objectives, such as $V_{100}^{\mathrm{PTV}}$, average dose to the organs-at-risk, and maximum dose to the PTV. To solve the non-convex subproblems that arise in their approach they use a heuristic method. ${ }^{46}$

Giantsoudi et al. ${ }^{20}$ have recently studied whether a model suggested by Wu et al. ${ }^{56}$ for IMRT could be successfully applied to HDR brachytherapy. In this model another evaluation measure, namely the gEUD, is optimized. To solve this model Giantsoudi et al. use the BFGS quasi-Newton method. Their results show that the model is suitable also for HDR brachytherapy, and the generated plans showed significant sparing of organs-atrisk compared to plans generated by a penalty-model.

Many models have also been developed for optimization of evaluation measures in external beam radiation. The survey by Ehrgott et al. ${ }^{17}$ present several of these. We present some of these results here as well. Deasy et al. ${ }^{15}$ have demonstrated that models that include constraints on dosimetric indices $\left(V_{x}^{y}\right.$ and $\left.D_{x}^{y}\right)$ are non-convex and that multiple local optima are possible. This implies that the inclusion of constraints on dosimetric indices leads to computational challenges, independent of the type of radiotherapy.

Zinchenko et al. ${ }^{58}$ present an approach for optimizing the DVH which uses the fact that any given DVH can be determined uniquely by an infinite sequence of generalized equivalent uniform dose values,

$$
\left\{\operatorname{gEUD}_{a}\right\}_{a=1,2,3, \ldots}
$$

By adding constraints on only a few of these gEUD $_{a}$ 's it is possible to obtain a convex approximation. However, choosing which of the gEUD $_{a}$ 's to constrain is hard, and probably not intuitive. Also Romeijn et al. ${ }^{45}$ introduce a way to approximate dosimetric indices. They use conditional value-at-risk as an approximation for D-measures, which yields a linear programming model. Hence the model can be solved easily to optimality.

\subsubsection{Optimization of catheter positioning}

It is well known that the placements of catheters is crucial for the resulting dose distribution. It is therefore valid to assume that optimization of their placement leads to a better dose distribution. The first attempts to optimize catheters positions that we know of were presented in 2009, when both Ayotte et al. ${ }^{3}$ and Karabis et al. ${ }^{28}$ presented heuristic methods for optimizing catheter positions.

The heuristic by Ayotte et al. is a constructive heuristic. It starts by solving the linear penalty model using a very high number of catheters. The heuristic then removes catheters based on the fraction of the total dwell time that is attributable to each catheter in the solution, and then resolves the linear penalty model. This is repeated until the desired number of catheters is reached. Their results show that compared to using a priori determined catheter positions the objective function value was improved, and that the doses to healthy tissue are reduced.

Karabis et al. present an optimization model for catheter positioning which is an extension of a penalty model. To integrate catheter positioning they introduce a set of feasible 
catheter positions, and then a binary variable (here $z$ is used) is assigned to each of these, in order to describe whether the position is used or not. A cardinality constraint that limits the number of catheters that can be used is added, and coupling constraints

$$
t_{j} \leq M z
$$

that ensure that a dwell point in a catheter is used only if that catheter is implanted. (Here $M$ is a sufficiently large number and $t_{j}$ is the time in a dwell point which is in the catheter that $z$ corresponds to.) This yields a mixed-integer nonlinear problem or a mixed-integer linear problem, depending on which type of penalty model that is used. To solve the model they try two approaches: standard optimization software and a heuristic. The standard software has troubles solving the model, and only succeeds when relatively few possible positions are introduced. Their heuristic is only sketchily described; it is a combination of simulated annealing and a scoring method for the binary part, and a quasi-Newton method for the continuous part. Also their results show that improved dose distributions are obtained when the catheter positioning is optimized.

Gorissen et al. ${ }^{21}$ also include catheter positioning in their model in the same way as Karabis et al. ${ }^{28}$. To enable for a standard optimization software to solve their model, they specify the constraints (3.4) as indicator constraints. They also add constraints that forbid using two adjacent catheter positions, that is, constraints of the type

$$
z_{1}+z_{2} \leq 1
$$

where catheters 1 and 2 are adjacent. This reduces the number of possible catheter positionings greatly, but it is of course a restriction of the original problem.

Siauw et al. ${ }^{52}$ present an approach that is quite different from the others. Their method consists of three major components. The first component generates a large set of candidate catheter positions. In the other approaches presented above the set of positions to choose among corresponds to the holes in the template used and all catheters where assumed to be inserted parallelly, this yields approximately 50 possible positions. Siauw et al. generate approximately 5000 possible positions, with different entry points and angles. The second component minimizes the number of catheters used under a constraint that all dose-calculation points shall be covered. A dose-calculation point is said to be covered if the distance to an implanted catheter is less than a given value. The third component optimizes the dose distribution within the chosen catheters, using the method by Siauw et al. ${ }^{51}$. Their results show that the catheter positions obtained with their method are satisfactory, but there is no guarantee that they are optimal with respect to the dose plan. 



\section{4}

\section{Contributions of the Thesis}

Earlier chapters have given an introduction to mathematical optimization in radiotherapy and also presented some different models and methods. This chapter outlines our contributions to this field by giving brief overviews of the appended papers in Part II and also presents some other minor contributions. Our contributions can be divided into three areas: properties of the linear penalty model, alternative models for optimizing the dwell time distribution, and methods for optimizing catheter positioning.

\subsection{Properties of the linear penalty model}

Since the linear penalty model is the model most commonly used in practice, it is essential to understand the properties of the dose plans generated by this model, and this is the main goal of Paper A. The linear penalty model is a linear optimization model, and hence an extreme point of the feasible set is optimal. (Alternative optima could exist but tests show that it is unlikely.) It is well known that the number of basic variables at an extreme point equals the number of constraints. The property investigated in Paper A is mainly how many variables of different types that can be basic variables at an (optimal) extreme point.

In the linear penalty model (the first version in Table 3.2) there are three types of variables: times $\left(t_{j}\right)$, penalties $\left(w_{i}^{s}\right)$, and slacks (denoted by $s_{i s}^{\min / \max }$ ), and $2 \sum_{s=1}^{S}\left|N_{s}\right|$ constraints. Consider a specific dose-calculation point, $\hat{\imath}$, in structure $\hat{s}$ and assume that the total dose to this point is $D o s e_{\hat{\imath}}^{\hat{s}}=\sum_{j=1}^{n} d_{\hat{\imath} j}^{\hat{s}} t_{j}$. The constraints associated with this dose-calculation point, written on standard form, are:

$$
\begin{aligned}
w_{\hat{\imath}}^{\hat{s}}-s_{\hat{\imath} \hat{s}}^{\min } & =M_{\min }^{\hat{s}}\left(D_{\min }^{\hat{s}}-\operatorname{Dos}_{\hat{\imath}}^{\hat{s}}\right) \\
w_{\hat{\imath}}^{\hat{s}}-s_{\hat{\imath} \hat{s}}^{\max } & =M_{\max }^{\hat{s}}\left(\operatorname{Dos}_{\hat{\imath}}^{\hat{s}}-D_{\max }^{\hat{s}}\right) \\
w_{\hat{\imath}}^{\hat{s}} & \geq 0 .
\end{aligned}
$$


Constraints 4.1 and 4.2 are equivalent to Constraints $3.2 \mathrm{a}$ and $3.2 \mathrm{~b}$, respectively. Depending on the value of $\operatorname{Dose}_{\hat{\imath}}^{\hat{s}}$ it is possible to deduce which variables that have to be basic, and Table 4.1 displays this for different cases. It is possible to illustrate this result as in Figure 4.1, where segments (1), (2) and (3) correspond to constraints 4.1, 4.2, and 4.3 respectively.

Now let $n_{d}$ be the number of dose-calculation points with $D o s e_{\hat{\imath}}^{\hat{s}}$ equal to $D_{\text {min }}^{\hat{s}}$ or $D_{\max }^{\hat{s}}$. Then the number of basic variables that are slacks or penalties is at least $2 \sum_{s} N_{s}-n_{d}$, and hence the maximum number of basic time variables is $n_{d}$. It is therefore possible to conclude that the total number of positive dwell times is limited by the number of dose-calculation points on their upper or lower dose limits.

As mentioned in Section 3.3.2, plans generated by the linear penalty model tend to be dominated by a few dwell positions with dwell times large compared to the others ${ }^{13}$. Given the result above this is not surprising since in practice relatively few values of Dose $e_{\hat{\imath}}^{\hat{s}}$ will equal $D_{\min }^{\hat{s}}$ or $D_{\max }^{\hat{s}}$, and hence only a few dwell time variables become non-zero (that is, basic). Some of these times then have to take large values in order to reach the desired overall dose level. The long dwell times observed in clinical practice can thus be explained by the distribution of basic variables, and this is the primary contribution of Paper A.

Paper B also considers a property of the linear penalty model, namely the relationship between dosimetric indices and the objective function used. Since physicians use dosimetric indices to evaluate dose plans it is of interest to see if there is a relationship between the values of these indices and the value of the linear penalty function. Paper B investigates this by generating a large number of dose plans. The results indicate that the connection between the objective function value and the values of a number of dosimetric indices is inadequate for some patients. Based on our results it seems as the objective function of the linear penalty model can roughly distinguish between better and worse dose plans, but that something is lacking in order to find the best dose plan. Hence the linear penalty model might work as a good tool for finding satisfactory dose plans, but those plans are not likely to be optimal with respect to the dosimetric indices which are used for their evaluation.

\subsection{Alternative models for optimizing the dwell time distribution}

Since the linear penalty model yields solutions with undesired properties, we have also presented two alternative models. The first model, which is given in Paper A, focuses on mitigating the long dwell times, and is an alternative penalty model. The second model, which is the one that we recommend, is presented in Paper C. It uses an approximation of the dosimetric indices and there is therefore a clear relationship between the model and the dosimetric indices.

\subsubsection{An alternative penalty model}

There are a few ways to allow more dwell time variables to be basic in the linear penalty model. One would be to generate many more dose-calculation points, thereby increasing 
Table 4.1: Number of forced basic variables from Constraints (4.1) and (4.2).

\begin{tabular}{ccc}
\hline \hline$D o s e_{\hat{i}}^{\hat{s}} \in$ & Basic variables & No. basic variables \\
\hline$\left[0, D_{\text {min }}^{\hat{s}}\right)$ & $w_{\hat{i}}^{\hat{s}}, s_{\hat{i} \hat{s}}^{\max }$ & 2 \\
{$\left[D_{\min }^{\hat{s}}\right]$} & $s_{\hat{i} \hat{s}}^{\max }$ & 1 \\
$\left(D_{\min }^{\hat{s}}, D_{\max }^{\hat{s}}\right)$ & $s_{\hat{i} \hat{s}}^{\min }, s_{\hat{i} \hat{s}}^{\max }$ & 2 \\
{$\left[D_{\max }^{\hat{s}}\right]$} & $s_{\hat{i} \hat{i}}^{\min }$ & 1 \\
$\left(D_{\max }^{\hat{s}}, \infty\right)$ & $w_{\hat{i}}^{\hat{s}}, s_{\hat{i} \hat{s}}^{\min }$ & 2 \\
\hline \hline
\end{tabular}

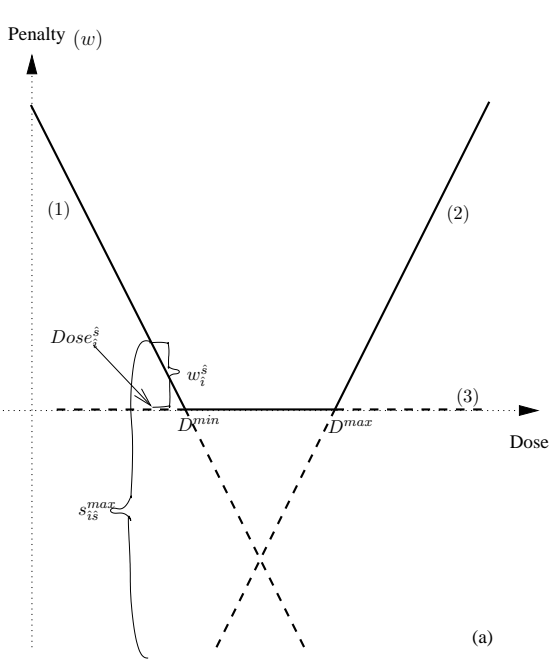

Penalty $(w)$

$\Lambda$
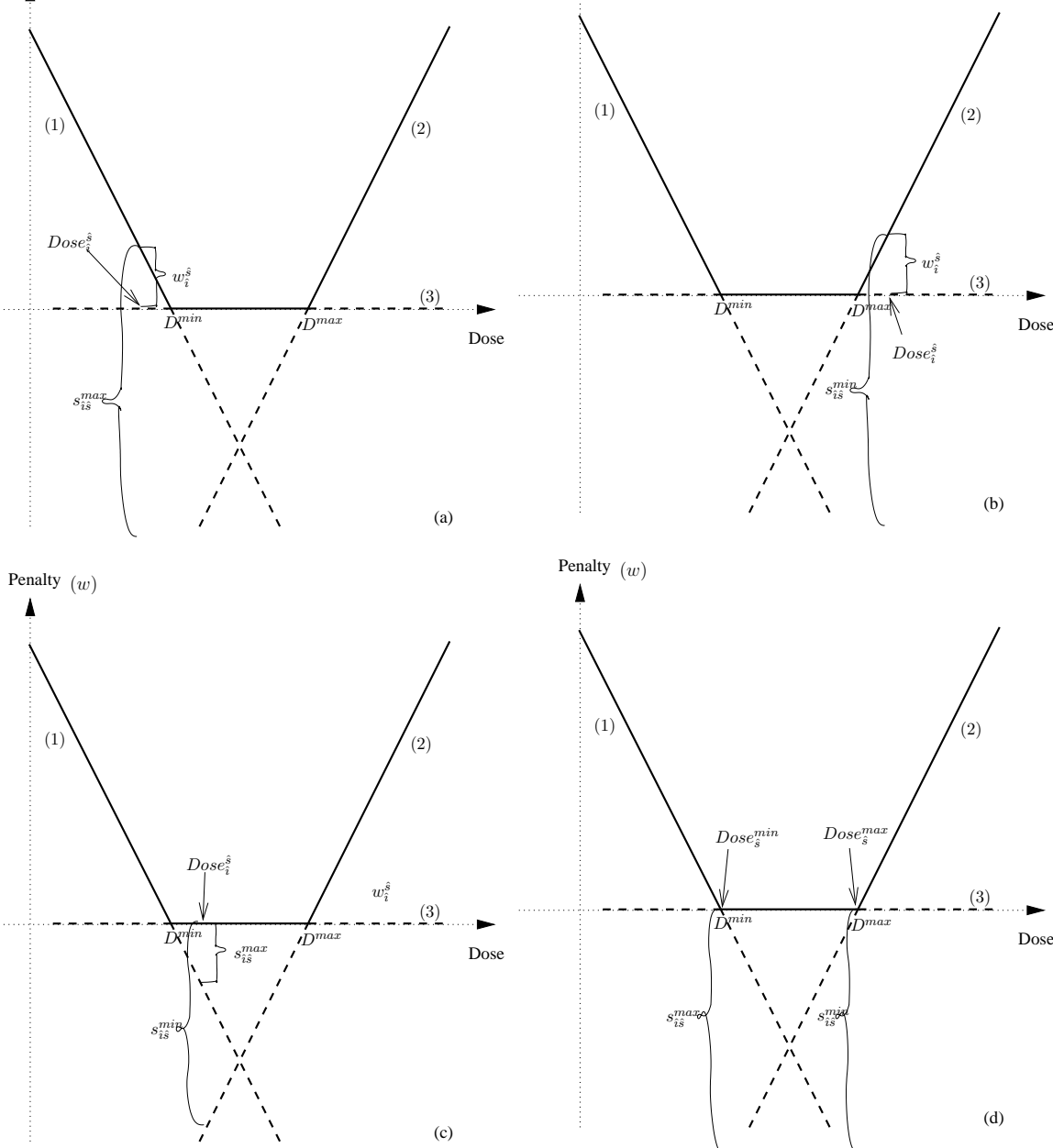

Penalty $(w)$

$\Lambda$

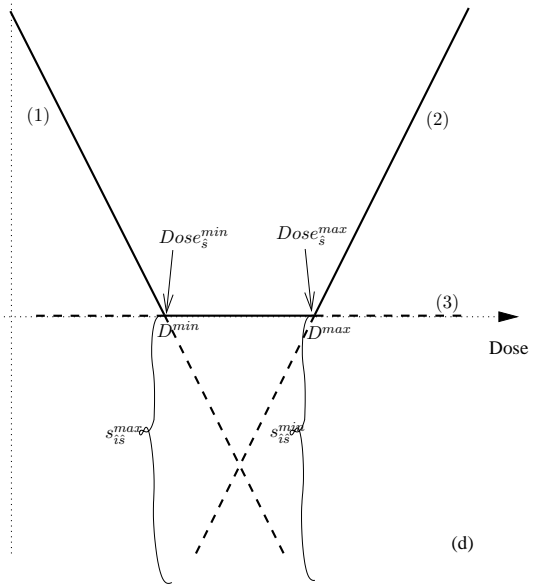

Figure 4.1: Illustration of the variables that have to be basic given the dose at dosecalculation point $\hat{\imath}$, in structure $\hat{s}$. 
the likelihood that dose-calculation points receive doses equal to their upper or lower bounds. In Paper A we instead propose that another type of penalties are used, namely convex piecewise linear penalties.

When using convex piecewise linear penalties more breakpoints are introduced, which increases the likelihood that the dose at a dose-calculation point coincides with the breakpoint. This allows more dwell times to be basic and hence positive. The piecewise linear penalties also enable a further differentiation of the penalties, e.g. points far from the required dose-range can receive a higher penalty than those closer to the interval.

The dose plans obtained by the linear penalty model and those from the alternative model yield quite similar dosimetric indices, and this indicates that the solutions are almost clinically equivalent. When comparing dwell times there is however a large difference, since the maximal dwell times are greatly reduced (average 30\%) and the numbers of active dwell positions are increased (average 20\%), implying a decrease in the undesired hot spots. Hence this model succeeds in mitigating the long dwell time, which was the goal.

\subsubsection{A model including dosimetric indices}

The alternative penalty function mitigated the problem with long dwell times but it did not help much with the relationship between the model and the dosimetric indices. Hence another model was needed. Several researchers have modeled the dosimetric indices exactly, as mentioned in Section 3.3.3, and drawn the conclusion that such models can not be solved exactly. They therefore construct methods for solving the model approximately. In Paper $\mathrm{C}$ we choose another approach instead: to approximate the dosimetric indices in a way that results in a model that can be solved to optimality within reasonable time. Our approximation utilises the similarity between $D_{x}^{y}$ and value-at-risk.

Value-at-risk (VaR) is a measure that is widely used to measure risk in financial mathematics. It assumes that there is a distribution that describes the risk of a certain portfolio. Based on this distribution, it defines a threshold value such that the probability of a loss that exceeds this value, over a certain time horizon, and for a given portfolio, is equal to a given probability. For example, if a portfolio has a weekly $10 \%$ VaR of $\$ 2000$, then there is a 0.10 probability that the portfolio will decrease in value by more than $\$ 2000$ over a week. It is well known that solving a model that includes constraints on VaR is very hard, and an alternative approach known as conditional value-at-risk (CVaR) has therefore been developed. The CVaR measures the expected loss of the $\alpha$ worst outcomes, where $\alpha$ is a given percentage.

The dosimetric index $D_{x}^{y}$ is actually equivalent to $\mathrm{VaR}$ for the dose distribution, and it is hence not surprising that exact dosimetric models are hard to solve. In Paper $\mathrm{C}$ we therefore propose that the CVaR of the dose distribution is used in the model as an approximation of $D_{x}^{y}$. The meaning of bounding (optimizing) CVaR is easy; instead of bounding (optimizing) the minimum dose to the highest receiving fraction, the mean dose to this fraction is bounded (optimized). It is also easy to include CVaR constraints (objectives) in an optimization model, since it only requires linear constraints (and objective) ${ }^{44}$.

Our studies of this dosimetric model show that it corresponds well with constraining dosimetric indices. Comparing the dose plans obtained by the linear penalty model to those from the dosimetric model, show that they are almost clinically equivalent. Hence 
the model proposed in Paper $\mathrm{C}$ is a viable surrogate for optimizing dosimetric indices. Below we present some theoretical results regarding the proposed model which are not included in Paper C.

\section{Mathematical notation}

For our theoretical analysis we need to express the CVaR measure mathematically in the context of dose distributions. The cumulative DVH for a structure $s$ of a dose distribution can formally be expressed as

$$
\operatorname{DVH}(\delta)=\frac{\left|\left\{i \in N_{s}: \operatorname{Dose}_{i}^{s} \geq \delta\right\}\right|}{\left|N_{s}\right|},
$$

where $N_{s}$ and Dose $e_{i}^{s}$ have the same meaning as in Table 3.1.

Definition 4.1. The Value-at-Risk of a dose distribution at a given level $\alpha \in(0,1)$ is the smallest dose level $\delta$ such that the percentage of the structure that recieves a dose above $\delta$ is no larger than $1-\alpha$. Formally,

$$
\operatorname{VaR}_{\alpha}=\min \{\delta \in \mathbb{R}: \operatorname{DVH}(\delta) \leq 1-\alpha\}
$$

Definition 4.2. The Conditional Value-at-Risk of a dose distribution at a given level $\alpha \in(0,1)$ is the mean of all doses that exceed $\operatorname{VaR}_{\alpha}$. Formally,

$$
\mathrm{CVaR}_{\alpha}=\operatorname{VaR}_{\alpha}+\frac{1}{(1-\alpha)\left|N_{s}\right|} \sum_{i \in N_{s}}\left(\text { Dose }_{i}^{s}-\mathrm{VaR}_{\alpha}\right)^{+},
$$

where $(x)^{+}=\max (0, x)$.

Example 4.1 shows how $\mathrm{CVaR}$ and VaR are calculated for a simple DVH, and gives a graphical interpretation.

So far we have only presented CVaR of the upper tail of the DVH. In financial applications this is the only tail considered since there is only a problem if the loss is too big, not if the profit is too big. However, for radiotherapy it is also important to consider the lower tail, since underdosage of a structure, especially the target volume, can be just as bad as overdosage. Therefore we similary define VaR and CVaR for the lower tail.

Definition 4.3. The lower tail Value-at-Risk of a dose distribution at a given level $\alpha \in$ $(0,1)$ is the largest dose level $\delta$ such that the percentage of the structure that recieves a dose below $\delta$ is no larger than $1-\alpha$. Formally,

$$
\operatorname{LVaR}_{\alpha}=\max \{\delta \in \mathbb{R}: \operatorname{DVH}(\delta) \geq 1-\alpha\} .
$$

Definition 4.4. The lower tail Conditional Value-at-Risk of a dose distribution at a given level $\alpha \in(0,1)$ is the mean of all doses that are less than $\operatorname{LVaR}_{\alpha}$. Formally,

$$
\operatorname{LCVaR}_{\alpha}=\operatorname{LVaR}_{\alpha}-\frac{1}{(1-\alpha)\left|N_{s}\right|} \sum_{i \in N_{s}}\left(\operatorname{LVaR}_{\alpha}-\operatorname{Dose}_{i}^{s}\right)^{+} .
$$

Rockafellar et al. ${ }^{44}$ have shown that when constraining (optimizing) CVaR it is possible to replace VaR with an auxiliary variable, and also each $\left(\operatorname{Dose}_{i}^{s}-\mathrm{VaR}_{\alpha}\right)^{+}$can be replaced by an auxiliary variable. Using this reformulation we can present a version of the optimization model we propose in Paper $\mathrm{C}$ in which CVaR for the dose distribution is both constrained and optimized. This version is found in Table 4.3 . 


\section{Example 4.1}

Consider an example with 20 dose-calculation points in a certain structure, with the dose to each of them given in Table 4.2. For this example VaR at $80 \%$ is the lowest dose such that four (20\% of 20 is four) dose-calculation points recieve a dose that is higher than VaR. Sorting the doses to all dose-calculation points we see that the five highest doses are $9.5,10,10,10.5$, and 11 . $\mathrm{VaR}_{0.8}$ is therefore $9.5+\epsilon$, where $\epsilon$ is an arbitrarily small number.

CVaR is the mean of all doses that exceeds $9.5+\epsilon$, hence it is the mean of the four highest doses, that is, $\frac{11+10+10.5+10}{4}=10.375 \mathrm{~Gy}$. The DVH corresponding to this example is given in Figure 4.2, where also $\mathrm{VaR}$ and $\mathrm{CVaR}$ are marked. The graphical interpretation of $\mathrm{CVaR}$ is that $\mathrm{CVaR}$ at level $\alpha \%$ is the area below the $\mathrm{DVH}$, and the horizontal line at $100-\alpha \%$, that is, the grey area in Figure 4.2, divided by $100-\alpha \%$ for normalization.

Table 4.2: The doses received by the 20 dose-calculation points in the structure.

$\begin{array}{lllllllllll}\text { Point } & 1 & 2 & 3 & 4 & 5 & 6 & 7 & 8 & 9 & 10 \\ \text { Dose (Gy) } & 9 & 8 & 9 & 9 & 8 & 9.5 & 11 & 10 & 9.5 & 9 \\ \text { Point } & 11 & 12 & 13 & 14 & 15 & 16 & 17 & 18 & 19 & 20 \\ \text { Dose (Gy) } & 9 & 10.5 & 7.5 & 10 & 9 & 8.5 & 9.5 & 9 & 9 & 9.5\end{array}$

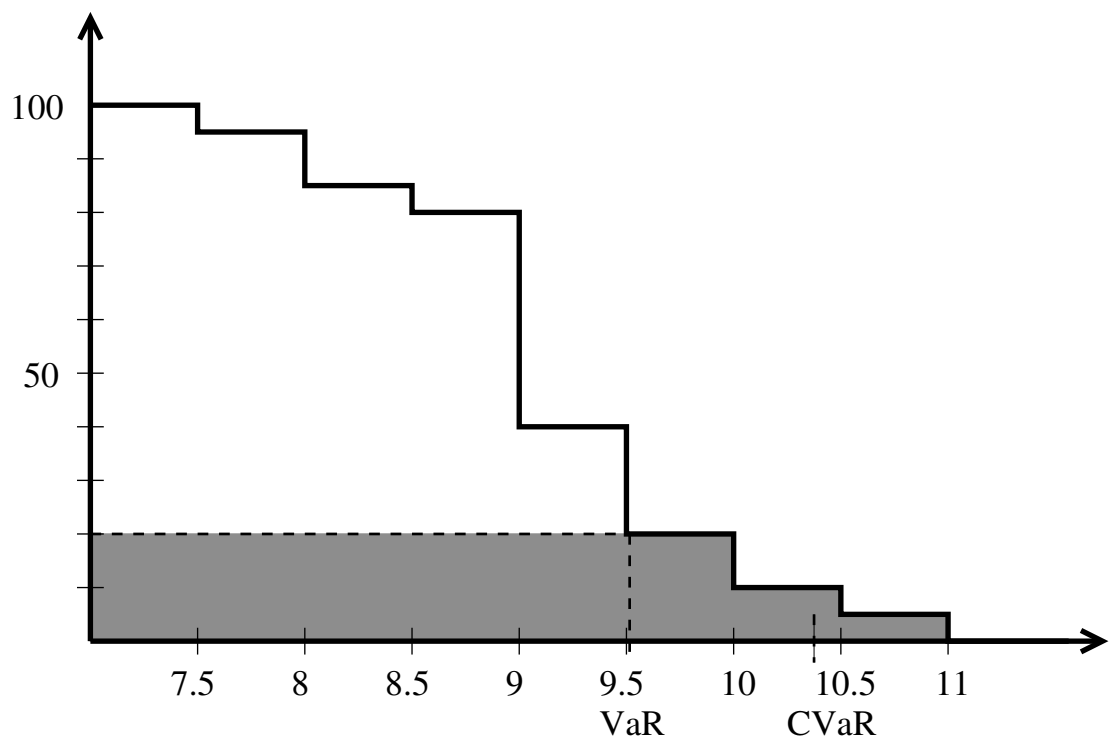

Figure 4.2: The DVH for the structure in Example 4.1, where VaR and CVaR are marked. 


\section{Parameters}

$S \quad=$ Set of structures.

$s_{o} \quad=$ Structure for which CVaR is optimized

$N_{s} \quad=$ Set of dose-calculation points in structure $s$.

$T=$ Set of dwell points.

$V^{s}=$ Set of CVaR the should be constrained for structure $s$.

$V L^{s}=$ Set of LCVaR the should be constrained for structure $s$.

$L_{k}^{s} \quad=$ Lower bound on LCVaR $k$ for structure $s$.

$U_{k}^{s} \quad=$ Upper bound on CVaR $k$ for structure $s$.

$\alpha_{k}^{s x}= \begin{cases}\text { if } x=u & \text { The } \alpha \text { of CVaR } k \text { for structure } s . \\ \text { if } x=l & \text { The } \alpha \text { of LCVaR } k \text { for structure } s . \\ \text { if } x=o & \text { The } \alpha \text { for LCVaR of the objective. }\end{cases}$

$d_{i j}^{s} \quad=$ Dose-rate contribution from dwell point $j$ to dose-calculation point $i$ in structure $s$.

\section{Variables}

$\operatorname{Dose}_{i}^{s}=$ Dose to dose-calculation point $i$ in structure $s$.

$v_{k}^{s x}= \begin{cases}\text { if } x=u & \text { Auxiliary variable for } \operatorname{VaR} k \text { for structure } s . \\ \text { if } x=l & \text { Auxiliary variable for LVaR } k \text { for structure } s . \\ \text { if } x=o & \text { Auxiliary variable for LVaR of the objective. }\end{cases}$

$\delta_{i k}^{s x}= \begin{cases}\text { if } x=u & \text { Auxiliary variable for } \operatorname{CVaR} k, \text { and dose-calculation point } i, \text { for structure } s . \\ \text { if } x=l & \text { Auxiliary variable for } \operatorname{LCVaR} k \text {, and dose-calculation point } i \text {, for structure } s . \\ \text { if } x=o & \text { Auxiliary variable for dose-calculation point } i \text { in the objective (structure } s_{o} \text { ). }\end{cases}$

$t_{j} \quad=$ Dwell time at point $j$.

Objective function

$$
\operatorname{minimize} \quad v^{s_{o} o}-\frac{1}{\left(1-\alpha^{s_{o} o}\right)\left|N_{s_{o}}\right|} \sum_{i \in N_{s_{o}}} \delta_{i}^{s_{o} o}
$$

Subject to

$$
\begin{array}{rlrl}
\operatorname{Dose}_{i}^{s} & =\sum_{j \in T} d_{i j}^{s} t_{j} & & \forall s \in S, i \in N_{s} \\
v_{k}^{s l}-\frac{1}{\left(1-\alpha_{k}^{s l}\right)\left|N_{s}\right|} \sum_{i \in N_{s}} \delta_{i k}^{s l} \geq L_{k}^{s} & & \forall s \in S, k \in V L_{s} \\
v_{k}^{s u}+\frac{1}{\left(1-\alpha_{k}^{s u}\right)\left|N_{s}\right|} \sum_{i \in N_{s}} \delta_{i k}^{s u} \leq U_{k}^{s} & & \forall s \in S, k \in V_{s} \\
v^{s_{o} o}-\operatorname{Dose}_{i}^{s_{o}} & \leq \delta_{i}^{s_{o} o} & & \forall i \in N_{s_{o}} \\
v_{k}^{s l}-\operatorname{Dose}_{i}^{s}-\leq \delta_{i k}^{s l} & & \forall s \in S, k \in V L_{s}, i \in N_{s} \\
\operatorname{Dose}_{i}^{s}-v_{k}^{s u} & \leq \delta_{i k}^{s u} & & \forall s \in S, k \in V_{s}, i \in N_{s} \\
\delta_{i k}^{s x} & \geq 0 & & \forall s \in S, k \in V(L)_{s}, i \in N_{s} \\
t_{j} & \geq 0 & & \forall j \in T
\end{array}
$$

Table 4.3: Our model for optimizing and constraining $C V a R$ and $L C V a R$. 


\section{Homogeneity}

In Paper $\mathrm{C}$ we choose to optimize homogeneity while constraining CVaR of the dose distribution. This requires formulating HI (defined in Equation 2.1) in a way that can be optimized. Doing this exactly yields a model that is as hard to solve as those that model dosimetric indices exactly; hence we propose an approximation for $\mathrm{HI}$ as well. In Paper C we present a short motivation of why our approximation should work. Below we instead present how we deduced it from CVaR.

Denote the prescription dose by $P$, and let $\alpha_{u}$ and $\alpha_{l}$ correspond to the $\alpha$ in VaR/CVaR and LVaR/LCVaR, respectively. Since $V_{150}^{s}$ is the precentage of the structure $s$ that recieves a dose above $150 \%$ of the prescription dose, that is, above $1.5 P$, we have that

$$
V_{150}^{s}=100\left(1-\alpha_{u}\right),
$$

when $\operatorname{VaR}_{\alpha_{u}}=1.5 P$. Similary for $V_{100}^{s}$ we have that

$$
V_{100}^{s}=100-100\left(1-\alpha_{l}\right),
$$

when $\mathrm{VaR}_{\alpha_{l}}=P$. Next note that the following expressions can be obtained from Equations 4.6 and 4.8 .

$$
\begin{aligned}
& \left(1-\alpha_{u}\right)=\frac{\sum_{i \in N_{s}}\left(\text { Dose }_{i}^{s}-\mathrm{VaR}_{\alpha_{u}}\right)^{+}}{\left|N_{s}\right|\left(\mathrm{CVaR}_{\alpha_{u}}-\mathrm{VaR}_{\alpha_{u}}\right)} \\
& \left(1-\alpha_{l}\right)=\frac{\sum_{i \in N_{s}}\left(\mathrm{LVaR}_{\alpha_{l}}-\operatorname{Dose}_{i}^{s}\right)^{+}}{\left|N_{s}\right|\left(\mathrm{LVaR}_{\alpha_{l}}-\mathrm{LCVaR}_{\alpha_{l}}\right)}
\end{aligned}
$$

Using this we know that

$$
\begin{gathered}
\max H I \Leftrightarrow \max \left(1-\frac{V_{150}^{\mathrm{CTV}}}{V_{100}^{\mathrm{CTV}}}\right) \Leftrightarrow \min \frac{V_{150}^{\mathrm{CTV}}}{V_{100}^{\mathrm{CTV}}} \Leftrightarrow \min \frac{1-\alpha_{u}}{1-\left(1-\alpha_{l}\right)} \Leftrightarrow \\
\Leftrightarrow \min \left(\frac{\sum_{i \in N_{\mathrm{CTV}}}\left(\operatorname{Dose}_{i}^{\mathrm{CTV}}-1.5 P\right)^{+}}{\left|N_{\mathrm{CTV}}\right|\left(\mathrm{CVaR}_{\alpha_{u}}-1.5 P\right)}\right) /\left(1-\frac{\sum_{i \in N_{\mathrm{CTV}}}\left(P-\operatorname{Dose}_{i}^{\mathrm{CTV}}\right)^{+}}{\left|N_{\mathrm{CTV}}\right|\left(P-\mathrm{LCVaR}_{\alpha_{l}}\right)}\right) \Leftrightarrow \\
\Leftrightarrow \min \frac{P-\mathrm{LCVaR}_{\alpha_{l}}}{\mathrm{CVaR}_{\alpha_{u}}-1.5 P} \cdot \frac{\sum_{i \in N_{\mathrm{CTV}}}\left(\operatorname{Dose}_{i}^{\mathrm{CTV}}-1.5 P\right)^{+}}{\left|N_{\mathrm{CTV}}\right|\left(P-\mathrm{LCVaR}_{\alpha_{l}}\right)-\sum_{i \in N_{\mathrm{CTV}}}\left(P-\text { Dose }_{i}^{\mathrm{CTV}}\right)^{+}} .
\end{gathered}
$$

To obtain an approximation of HI we assume that the first fraction of Equation 4.14 is constant. It is unlikely that this is true, but it is likely that the variation of the first fraction is quite small compared to that of the second fraction. Almost all parameters of the second fraction can easily be incorporated into an optimization model. The exception is $P-\mathrm{LCVaR}_{\alpha_{l}}$ which we replace with a parameter $\Upsilon$. This yields the fractional linear objective function

$$
\min \frac{\sum_{i \in N_{\mathrm{CTV}}}\left(\sum_{j \in T} d_{i j}^{\mathrm{CTV}} t_{j}-1.5 P\right)^{+}}{\left|N_{\mathrm{CTV}}\right| \Upsilon-\sum_{i \in N_{\mathrm{CTV}}}\left(P-\sum_{j \in T} d_{i j}^{\mathrm{CTV}} t_{j}\right)^{+}}
$$


which we use in our model in Paper $\mathrm{C}$ as a surrogate for maximization of HI. We assume that $\Upsilon$ is chosen so that the denominator is $>0$ for all feasible $t$.

Optimization models that contain linear constraints and a fractional linear objective can be solved easily, since they can be transformed into linear models with the help of one auxiliary variable. We do this transformation for our model in the appendix of Paper C.

\section{Convexity of our homogeneity approximation}

To investigate which solution methods that could be used to solve the model in Paper C it is necessary to know some properties of the objective function, such as whether it is convex or not. Below we show that objective function 4.15 is quasiconvex, but neither convex, concave nor quasiconcave.

It is easy to deduce that the numerator of the objective function is convex and that the denominator is concave. This does however not say anything about the convexity properties of the entire function. Example 4.2 shows that the objective function is neither convex nor concave in the general case.

\section{Example 4.2}

Consider a small example with two dose-calculation points and one dwell position. For one of the points the dose-rate contribution from the dwell position is 1 , and for the other it is 15 , while the prescription dose is 1 . Then the objective as a function of the dwell time is

$$
f(t)=\frac{(t-1.5)^{+}+(15 t-1.5)^{+}}{2 \Upsilon-(1-t)^{+}-(1-15 t)^{+}}
$$

Calculating the objective values for four different values of $t$ gives the following

$$
f(0)=0, \quad f(0.5)=\frac{6}{2 \Upsilon-\frac{1}{2}}, \quad f(1)=\frac{27}{4 \Upsilon}, \quad f(2)=\frac{29}{2 \Upsilon} .
$$

The function is convex if for all $0 \leq \lambda \leq 1$ we have that

$$
f\left(\lambda t_{1}+(1-\lambda) t_{2}\right) \leq \lambda f\left(t_{1}\right)+(1-\lambda) f\left(t_{2}\right)
$$

and concave if $\leq$ is replaced by $\geq$. Use $t_{1}=0$ and $t_{2}=2$, and two values of $\lambda, 0.5$ and 0.75 .

$$
\begin{aligned}
\lambda=0.5: \quad & f(0.5 \cdot 0+0.5 \cdot 2)=f(1)=\frac{27}{4 \Upsilon}<0.5 \cdot \frac{29}{2 \Upsilon}= \\
& =0.5 f(0)+0.5 f(2) \Rightarrow f \text { is not concave } \\
\lambda=0.75: \quad & f(0.75 \cdot 0+0.25 \cdot 2)=f(0.5)=\frac{6}{2 \Upsilon-\frac{1}{2}}>\left[\text { if } \Upsilon<\frac{29}{20}\right]> \\
& >0.25 \cdot \frac{29}{2 \Upsilon}=0.75 f(0)+0.25 f(2) \Rightarrow f \text { is not convex }
\end{aligned}
$$


Showing that the objective is neither convex nor concave, leaves the question of whether the function is quasiconvex or quasiconcave.

Definition 4.5. A function is quasiconvex if it for all $t^{1}, t^{2}$ and $0 \leq \lambda \leq 1$ holds that $f\left(\lambda t^{1}+(1-\lambda) t^{2}\right) \leq \max \left(f\left(t^{1}\right), f\left(t^{2}\right)\right)$, and quasiconvex if $\leq$ and max is replaced by $\geq$ and $\min$.

Below follows a proof that the objective is quasiconvex, and Example 4.3 provides a small counterexample to show that the objective is not quasiconcave.

\section{Lemma 4.1}

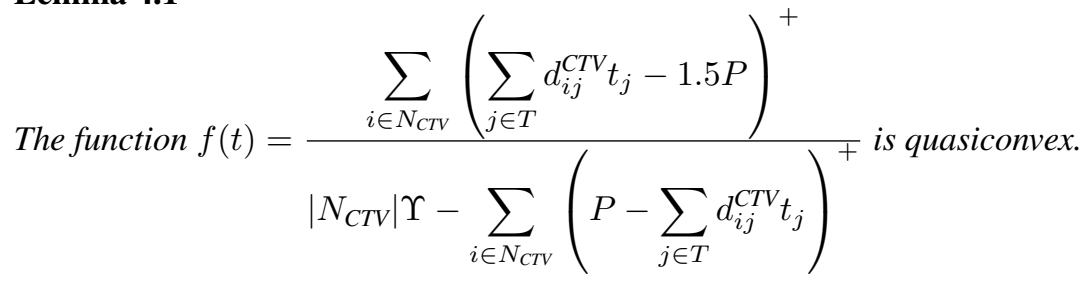

Proof: To simplify the proof the following notation is used

$g(t)=\sum_{i \in N_{\mathrm{CTV}}}\left(\sum_{j \in T} d_{i j}^{\mathrm{CTV}} t_{j}-1.5 P\right)^{+}$and $h(t)=\left|N_{\mathrm{CTV}}\right| \Upsilon-\sum_{i \in N_{\mathrm{CTV}}}\left(P-\sum_{j \in T} d_{i j}^{\mathrm{CTV}} t_{j}\right)^{+}$.

Choose two arbitrary points $t^{1}$ and $t^{2}$, and assume without loss of generality that $f\left(t^{1}\right) \geq$ $f\left(t^{2}\right)$. From this follows that

$$
\frac{g\left(t^{1}\right)}{h\left(t^{1}\right)} \geq \frac{g\left(t^{2}\right)}{h\left(t^{2}\right)} \Leftrightarrow g\left(t^{1}\right) h\left(t^{2}\right) \geq g\left(t^{2}\right) h\left(t^{1}\right) .
$$

Then the definition is used to prove quasiconvexity.

$$
\begin{aligned}
f\left(\lambda t^{1}+(1-\lambda) t^{2}\right) & =\frac{g\left(\lambda t^{1}+(1-\lambda) t^{2}\right)}{h\left(\lambda t^{1}+(1-\lambda) t^{2}\right)} \leq[g \text { is convex }] \leq \\
& \leq \frac{\lambda g\left(t^{1}\right)+(1-\lambda) g\left(t^{2}\right)}{h\left(\lambda t^{1}+(1-\lambda) t^{2}\right)} \leq[h \text { is concave and strictly positive }] \leq \\
& \leq \frac{\lambda g\left(t^{1}\right)+(1-\lambda) g\left(t^{2}\right)}{\lambda h\left(t^{1}\right)+(1-\lambda) h\left(t^{2}\right)}=\left[\text { Multiply with } h\left(t^{1}\right)\right]= \\
& =\frac{\lambda g\left(t^{1}\right) h\left(t^{1}\right)+(1-\lambda) g\left(t^{2}\right) h\left(t^{1}\right)}{\lambda h\left(t^{1}\right) h\left(t^{1}\right)+(1-\lambda) h\left(t^{1}\right) h\left(t^{2}\right)} \leq[\text { Use } 4.16] \leq \\
& \leq \frac{\lambda g\left(t^{1}\right) h\left(t^{1}\right)+(1-\lambda) g\left(t^{1}\right) h\left(t^{2}\right)}{\lambda h\left(t^{1}\right) h\left(t^{1}\right)+(1-\lambda) h\left(t^{1}\right) h\left(t^{2}\right)}= \\
& =\frac{g\left(t^{1}\right)}{h\left(t^{1}\right)} \cdot \frac{\lambda h\left(t^{1}\right)+(1-\lambda) h\left(t^{2}\right)}{\lambda h\left(t^{1}\right)+(1-\lambda) h\left(t^{2}\right)}= \\
& =\frac{g\left(t^{1}\right)}{h\left(t^{1}\right)}=f\left(t^{1}\right)=\max \left(f\left(t^{1}\right), f\left(t^{2}\right)\right) .
\end{aligned}
$$




\section{Example 4.3}

Introduce two dwell positions ( $t_{1}$ and $\left.t_{2}\right)$ and two dose-calculation points, with dose rate contributions given by the matrix $\left(\begin{array}{ll}1 & 0 \\ 0 & 1\end{array}\right)$. With prescription dose 1 , the objective as a function of the dwell times is

$$
f(t)=\frac{\left(t_{1}-1.5\right)^{+}+\left(t_{2}-1.5\right)^{+}}{2 \Upsilon-\left(1-t_{1}\right)^{+}-\left(1-t_{2}\right)^{+}}
$$

Calculating the objective for three different dwell times gives the following values.

$$
f(0,2)=\frac{0.5}{2 \Upsilon-1}, \quad f(2,0)=\frac{0.5}{2 \Upsilon-1}, \quad f(1,1)=0
$$

Since $f(1,1)<\min (f(2,0), f(0,2))$, and $(1,1)=\frac{1}{2}(2,0)+\frac{1}{2}(0,2)$, it follows that $f(t)$ is not quasiconcave.

\subsubsection{Theoretical comparison of the linear penalty model and our dosimetric model}

Both our dosimetric model in Table 4.3 and the linear penaly model in Table 3.2 are linear optimization problems. One migth therefore wonder how similar they are. Below we will use various transformations of the two models to compare them.

We start by considering the homogeneity objective (Equation 4.15). Assume that the optimal objective function value is $H^{*}$. Then the optimal solution of the homogeneity objective is optimal also in

$\min \sum_{i \in N_{\mathrm{CTV}}}\left(\sum_{j \in T} d_{i j}^{\mathrm{CTV}} t_{j}-1.5 P\right)^{+}-H^{*}\left(\left|N_{\mathrm{CTV}}\right| \Upsilon-\sum_{i \in N_{\mathrm{CTV}}}\left(P-\sum_{j \in T} d_{i j}^{\mathrm{CTV}} t_{j}\right)^{+}\right)$.

To see this consider the objective $\min \frac{g(t)}{h(t)}$, where $h(t)>0$. Assume that the optimal solution is $t^{*}$ with objective value $k$, then $g\left(t^{*}\right)-k h\left(t^{*}\right)=0$. Now, assume that there is a feasible $\hat{t}$ such that $g(\hat{t})-k h(\hat{t})<0$. Then $\frac{g(\hat{t})}{h(\hat{t})}<k$, contradicting that $t^{*}$ is the optimal solution, and hence $\hat{t}$ can not exist. The optimal value of $\min g(t)-k h(t)$ is hence greater or equal to zero, and since $g\left(t^{*}\right)-k h\left(t^{*}\right)=0$ we know that $t^{*}$ is an optimal solution of $\min g(t)-k h(t)$.

Equation 4.17 is equivalent to

$$
\min \sum_{i \in N_{\mathrm{CTV}}}\left(\sum_{j \in T} d_{i j}^{\mathrm{CTV}} t_{j}-1.5 P\right)^{+}-H^{*} \sum_{i \in N_{\mathrm{CTV}}}\left(P-\sum_{j \in T} d_{i j}^{\mathrm{CTV}} t_{j}\right)^{+}
$$

since constants can be removed from the objective without affecting the optimal solution. 
This objective function can be interpreted as an objective of the linear penalty model with

$$
\begin{aligned}
M_{\text {min }}^{\mathrm{CTV}} & =1, \\
M_{\text {max }}^{\mathrm{CTV}} & =H^{*}, \\
D_{\text {min }}^{\mathrm{CTV}} & =P, \\
D_{\max }^{\mathrm{CTV}} & =1.5 P .
\end{aligned}
$$

Hence the homogeneity objective and the linear penalty objective are closely related. However, there is a big difference in the interpretation of the parameters, since our parameters have a clear connection to $\mathrm{HI}$, unlike the parameters of the linear penalty model. It is also important to remember that the objective function of the linear penalty model also includes the OAR:s, and that this model does not include any dosimetric constraints.

Let us next consider how the linear penalty model corresponds to models including dosimetric indices. It is usually desired to maximize dosimetric indices related to the lower tail and minimize dosimetric indices related to the upper tail. Hence a weighted objective of dosimetric indices would look something like

$$
\max \sum_{s \in N_{s}}\left(\sum_{l \in V_{-}^{s}} w_{l}^{s} \mathbf{V}_{l}^{s}-\sum_{u \in V_{+}^{s}} w_{u}^{s} \mathbf{V}_{u}^{s}\right),
$$

where all weights $\left(w_{l / u}^{s}\right)$ are positive, and $V_{-}^{s}$ and $V_{+}^{s}$ are the sets of dosimetric indices that should be minimized and maximized, respectively, for structure $s$. Using that $\mathbf{V}_{l}^{s}=$ $100-100\left(1-\alpha_{l}\right), \forall l \in V_{-}^{s}$, and that $\mathbf{V}_{u}^{s}=100\left(1-\alpha_{u}\right), \forall u \in V_{+}^{s}$, we see that Objective function 4.18 is equivalent to

$$
\min \sum_{s \in S}\left(\sum_{l \in V_{-}^{s}} w_{l}^{s}\left(1-\alpha_{l}\right)+\sum_{u \in V_{+}^{s}} w_{u}^{s}\left(1-\alpha_{u}\right)\right) .
$$

Now Equations 4.12 and 4.13 can be used to show that Objective function 4.19 is equivalent to

$$
\begin{aligned}
\min \sum_{s \in S} & \left(\sum_{l \in V_{-}^{s}}\left(\frac{w_{l}^{s}}{\left|N_{s}\right|\left(\mathrm{LVaR}_{\alpha_{l}}-\mathrm{LCVaR}_{\alpha_{l}}\right)} \sum_{i \in N_{s}}\left(\operatorname{LVaR}_{\alpha_{l}}-\operatorname{Dose}_{i}^{s}\right)^{+}\right)\right. \\
& \left.+\sum_{u \in V_{+}^{s}}\left(\frac{w_{u}^{s}}{\left|N_{s}\right|\left(\mathrm{CVaR}_{\alpha_{u}}-\mathrm{VaR}_{\alpha_{u}}\right)} \sum_{i \in N_{s}}\left(\operatorname{Dose}_{i}^{s}-\mathrm{VaR}_{\alpha_{u}}\right)^{+}\right)\right) .
\end{aligned}
$$

This objective can now easily be compared to that of the linear penalty model. Doing this comparision we see that

$$
\begin{aligned}
M_{\text {min }}^{s} & =\frac{w_{l}^{s}}{\left(\mathrm{LVaR}_{\alpha_{l}}-\mathrm{LCVaR}_{\alpha_{l}}\right)}, \\
M_{\text {max }}^{s} & =\frac{w_{u}^{s}}{\left(\mathrm{CVaR}_{\alpha_{u}}-\mathrm{VaR}_{\alpha_{u}}\right)}, \\
D_{\text {min }}^{s} & =\mathrm{LVaR}_{\alpha_{l}}, \\
D_{\text {max }}^{s} & =\operatorname{VaR}_{\alpha_{u}} .
\end{aligned}
$$


Hence if the goal is to find an optimal dose plan with respect to the weighted objective of dosimetric indices, then the weights $\left(M_{\min / \max }^{s}\right)$ in the linear penalty model should correspond to the weights for the dosimetric indices considered divided with the difference between CVaR/LCVaR and VaR/LVaR, and the breakpoints should correspond to the $\mathrm{VaR} / \mathrm{LVaR}$. Hence it is possible to interpret the linear penalty model in dosimetric terms, but the penalties and breakpoints need to be chosen in a very specific way. This explain why the linear penalty model works quite well in clinical practice, but also why it is so hard to choose proper penalty parameters.

\subsection{Methods for optimization of the catheter positioning}

Several approaches for optimizing the catheter positioning have been proposed during the last few years, as mentioned in Section 3.3.4. In Paper D we develop an optimization model for catheter positioning. This model is based on the linear penalty model and includes catheter placement by introducing a set of feasible catheter positions and assigning to each position a binary variable that describes if it is used or not. This model is very similar to those by Karabis et al. ${ }^{28}$ and Gorissen et al. ${ }^{21}$.

Most models for dwell time distribution optimization can be extended to include catheter positioning by adding binary variables in the way described in Section 3.3.4. Table 4.4 presents the parameters, variables, and constraints that are needed to do this.

It seems that the resulting model is computationally demanding to solve, independent of which dwell time distribution optimization model that is extended to include catheter positioning. There is therefore a need to develop tailored methods that facilitate the integrated optimization of catheter positioning and dwell time distribution. In this section we present some work to this end.

\subsubsection{Using commercial software}

As a first step towards solving the cather optimization problem we investigated if it would be possible to use a commercial software, CPLEX 10.0. We investigated if tuning CPLEX or adding additional constraints could improve the solutions found when trying to solve the model. What we noticed was that when the generation of cuts was turned of, the solutions found improved (see Table 4.5), which implies that the standard integer cuts do not work well for the given formulation. It might therefore be interesting to investigate if it is possible to find specialised cuts.

We also considered adding some restricting inequalities that in practice are likely to hold for good solutions. Our inequalities limited the number of catheters used in subvolumes. They are reasonable because if many catheters are used in one subvolume there will not be enough catheters left to cover the rest of the treatment volume. Since the possible catheter positions we used in our tests correspond to the possible positions of the template they formed a square grid. The inequalities added (referred to as subcuts) limited the number of catheters used in each square to two. The subcuts are illustrated in Figure 4.3 and they are mathematically described by creating a set $S q_{i}$ for each square $i$, 


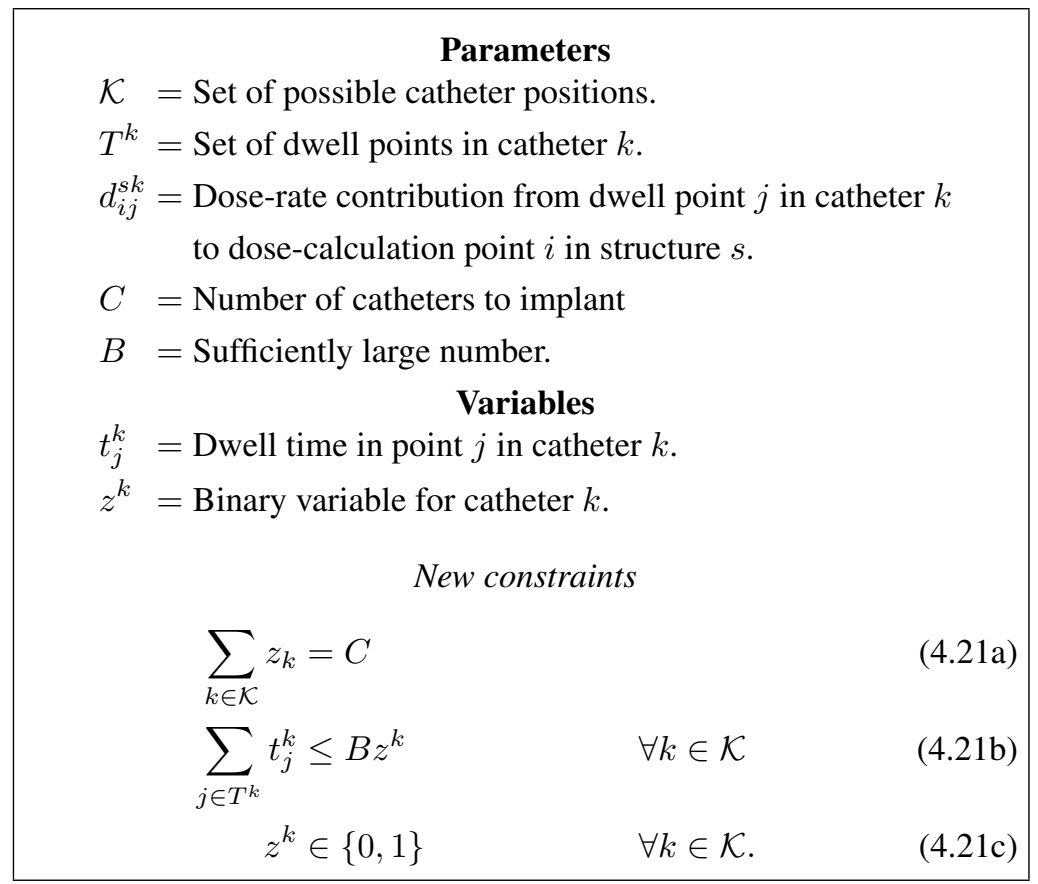

Table 4.4: The parameters, variables, and constraints needed to extend a dwell time distribution optimization model to also include optimization of the catehter positioning.

containing the catheters in the square and then restricting the $z$-sum for each square:

$$
\sum_{k \in S q_{i}} z_{k} \leq 2 \quad \forall i .
$$

Tests show that these subcuts support finding better solutions (see Table 4.5) even though they restrict the feasible space. In Paper D we choose not to include the subcuts in the model since we wanted to avoid unnecessary restrictions, but we turned off the generation of cuts in CPLEX since this improve the solutions found, as mentioned above.

\subsubsection{Heuristics}

In Paper D we develop three heuristics for the linear penalty model where catheter positioning has been integrated: a tabu search, a variable neighbourhood search, and a genetic algorithm. Out of the developed heuristics, variable neighbourhood search is clearly the best, outperforming the other heuristics in all test cases and a state-of-the-art optimization software (CPLEX) in most cases. The variable neighbourhood search heuristic utilise three kinds of neighbourhoods:

Neighbourhood 1: Move one catheter within a subset $\bar{R}$ of $\mathcal{K}$, or, mathematically, $K_{2} \in$ $N\left(K_{1}\right)$ if $\left(\left|K_{1} \cap K_{2}\right|=C-1\right) \wedge\left(K_{1} \triangle K_{2} \subseteq \bar{R}\right)$. The set $\bar{R}$ is generated uniformly 


\begin{tabular}{|c|cc|}
\hline Settings & Lower bound & Upper bound \\
\hline Standard & 133 & 228 \\
No cuts & 134 & 214 \\
No cuts+subcuts & 194 & 203 \\
\hline
\end{tabular}

Table 4.5: Runs of 15 hour with CPLEX (10.0) using different settings (cuts or not) and our restricting inequalities subcuts for one patient.

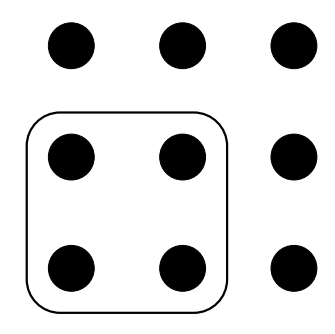

Figure 4.3: The subcuts limit the number of used catheters of those within the box to at most two.

randomly in each iteration as to include both used and unused catheters.

Neighbourhood 2: Move one catheter, or, mathematically, $K_{2} \in N\left(K_{1}\right)$ if $\left(\left|K_{1} \cap K_{2}\right|=\right.$ $C-1) \wedge\left(K_{1} \triangle K_{2} \subseteq \mathcal{K}\right)$.

Neighbourhood 3: As neighbourhood 1, but 2 catheters are to be moved, that is, mathematically, $K_{2} \in N\left(K_{1}\right)$ if $\left(\left|K_{1} \cap K_{2}\right|=C-2\right) \wedge\left(K_{1} \triangle K_{2} \subseteq \bar{R}\right)$.

Here $K_{1}$ and $K_{2}$ are two solutions, $N\left(K_{1}\right)$ are the neighbours of $K_{1}$, and $\triangle$ is the symmetric difference. A pseudo-code for the variable neighbourhood search can be found in Table 4.6. The best neighbour is found by solving an integer problem, since an explicit enumeration of all neighbours is more time consuming. The integer problem that is solved is similar to the original model but with additional constraints. With these additional constraints the problem can be solved to optimality fairly quickly.

The tabu search performs quite evenly with CPLEX given equal time, and the genetic algorithm performs quite poorly, around 50\% worse than CPLEX. The most interesting result from Paper D is however probably the improvement in objective value achieved when integrating catheter positioning in the optimization, compared to being restricted to the positions used in clinical practice; the mean improvement was 53\%.

\subsubsection{Branching rules}

In Paper E we develop the foundation for a branch-and-bound method that is adapted to the problem of finding an optimal dwell time distribution and catheter positioning. It is based on using a relaxation where Constraint $4.21 \mathrm{~b}$ has been removed. This constraint is then enforced in the branch-and-bound algorithm by a specialised branching rule that consider groups $\left(G_{l}\right)$ of $z_{k}$ variables which are geometrically close. Given an optimal solution $(\bar{t}, \bar{z})$ of the relaxation that does not satisfy Constraint $4.21 \mathrm{~b}$ it is possible to find a group $G_{L}$ that contains at least one $z_{k}$ that does not satisfy the constraint. Based on this group three branches are created.

Branch 1 All $z_{k}$ and $t_{j}^{k}$ in the group that violate Constraint $4.21 \mathrm{~b}$ are set to zero, and the total number of $z_{k}$ used in the group can not increase. This is enforced by adding 
solution=generate a random solution currentNeighbourhood $=1$

counter $=0$

while not fulfilled stopping criterion do

newSolution= best neighbour of solution in the currentNeighbourhood

if newSolution is better than solution then

currentNeighbourhood $=1$

solution=newSolution

counter $=0$

else

counter=counter +1

if counter $>$ counterLimit(currentNeighbourhood) then

currentNeighbour=currentNeighbour +1

counter $=0$

if currentNeighbour $>3$ then

Perturb solution randomly

currentNeighbour $=1$

\section{end if}

end if

end if

end while

Table 4.6: Pseudo-code for the variable neighbourhood search developed in Paper D.

the following constraints to the relaxation.

$$
\begin{aligned}
z_{k} & =0 & \forall k \in V \\
\sum_{j \in T_{k}} t_{j}^{k} & =0 & \forall k \in V \\
\sum_{k \in F} z_{k} & \leq\left\lceil\sum_{k \in G_{L}} \overline{z_{k}}\right\rceil &
\end{aligned}
$$

Branch 2 One of the $z_{k}$ in the group that violates Constraint $4.21 \mathrm{~b}$ must be used, and the total number of $z_{k}$ used in the group can not increase. This is enforced by adding the following constraints to the relaxation.

$$
\begin{aligned}
1 & \leq \sum_{k \in V} z_{k} \\
\sum_{k \in G_{L}} z_{k} & \leq\left\lceil\sum_{k \in G_{L}} \overline{z_{k}}\right\rceil
\end{aligned}
$$

Branch 3 The sum of $z_{k}$ in the group must increase by at least one. This is enforced by 
adding the following constraint to the relaxation.

$$
\sum_{k \in G_{L}} z_{k} \geq\left\lceil\sum_{k \in G_{L}} \overline{z_{k}}\right\rceil+1
$$

Here

$$
\begin{aligned}
& V=\left\{k \in G_{L}:\left(\overline{z_{k}}=0\right) \wedge\left(\sum_{j \in T_{k}} \overline{t_{j}^{k}}>0\right)\right\} \\
& \text { and } \\
& F=\left\{k \in G_{L}:\left(\overline{z_{k}}>0\right) \vee\left(\sum_{j \in T_{k}} \overline{t_{j}^{k}}=0\right)\right\} .
\end{aligned}
$$

One major advantage of this branch-and-bound version is that the relaxation separates into two subproblems. Our tests also showed that the dual bound improved faster than with a standard branch-and-bound method, if the groups formed a partition of all catheters. This tailored branch-and-bound is however far from solving the problem in a clinically acceptable time frame, and further research is therefore necessary.

\subsection{Future research}

Papers A and B clearly show that the linear penalty model is inadequate for finding optimal dose distributions. An alternative model should therefore be adapted in clinical practice. To facilitate this further research is needed. Our model presented in Paper C and the other models presented in Section 3.3.3 need to be thoroughly compared and evaluated to find which of them that is most suitable for clinical use. For the chosen model it is also important to develop class solutions for the parameters of the model. Most of the models focus on HDR brachytherapy for the prostate and validation for other treatment sites are therefore also needed.

Today ${ }^{192} \mathrm{Ir}$ is usually used for the treatment, but it would be possible to use other sources or combine different sources. Also, recently suggested are electronic x-ray sources that can vary the source energy, ${ }^{43}$ which in turn leads to different dose rates. This new possibility needs to be included in the optimization models to investigate whether it would be beneficial or not to use different energies.

Papers D and E studied methods for optimization of catheter positions. The heuristics of Paper D might be possible to use as they are in clinical practice. The exact approach presented in Paper E is still to time-consuming and further work is therefore needed, possibly by constructing tailor-made heuristics and node selection rules. 



\section{Bibliography}

[1] Alberts, B. (2004). Essential cell biology. Second edition. Garland science, New York.

[2] Alterovitz, R., Lessard, E., Pouliot, J., Hsu, I.-C. J., O’Brien, J. F., and Goldberg, K. (2006). Optimization of HDR brachytherapy dose distributions using linear programming with penalty costs. Medical Physics, 33(11):4012-4019.

[3] Ayotte, G., D’Amours, M., Aubin, S., Lessard, E., Pouliot, J., and Beaulieu, L. (2009). Sci-Thurs AM: YIS-02: Optimizing number and position of catheters within inverse planning simulated annealing (IPSA) for prostate and breast high dose rate brachytherapy. Medical Physics, 36(9):4315-4315.

[4] Bahr, G. K., Kereiakes, J. G., Horwitz, H., Finney, R., Galvin, J., and Goode, K. (1968). The Method of Linear Programming Applied to Radiation Treatment Planning. Radiology, 91(4):686-93.

[5] Baltas, D., Kolotas, C., Geramani, K., Mould, R. F., Ioannidis, G., Kekchidi, M., and Zampoglou, N. (1998). A conformal index (COIN) to evaluate implant qualityand dose specification in brachytheray. International Journal of Radiation Oncology*Biology*Physics, 40(2):515-524.

[6] Baltas, D., Katsilieri, Z., Kefala, V., Papaioannou, S., Karabis, A., Mavroidis, P., and Zamboglou, N. (2009). Influence of modulation restriction in inverse optimization with HIPO of prostate implants on plan quality: Analysis using dosimetric and radiobiological indices. IFMBE Proceedings, 25: 283-286.

[7] Beliën, J., Colpaert, J., De Boeck, L., and Demeulemeester, E. (2009). A Hybrid Simulated Annealing Linear Programming Approach for Treatment Planning in HDR Brachytherapy with Dose Volume Constraints. In Proceedings of the 35ORAHS; Leuven, Belgium; 12-17 July 2009. 
[8] Berglund, E. and Jönsson, B. (2007). Medicinsk fysik. Studentlitteratur, Lund.

[9] Beyzadeoglu, M., Beyzadeoglu, M., Özyigit, G., and Ebruli, C. (2010). Basic radiation oncology. Springer, Berlin.

[10] Boyle, P. and Levin, B. (2008). World cancer report 2008. International Agency for Research on Cancer, Lyon.

[11] Campbell, M. K. and Farrell, S. O. (2009). Biochemistry. Sixth edition. Thomson Brooks/Cole, Belmont.

[12] Cancerfonden (2011). Prostatacancer. http: / / www . cancerfonden. se/sv/ cancer/Cancersjukdomar/prostatacancer/.

[13] Chajon, E., Dumas, I., Touleimat, M., Magné, N., Coulot, J., Verstraet, R., Lefkopoulos, D., and Haie-Meder, C. (2007). Inverse planning approach for 3-D MRI-based pulse-dose rate intracavitary brachytherapy in cervix cancer. International Journal of Radiation Oncology*Biology*Physics, 69(3):955-961.

[14] De Boeck, L., Beliën, J., and Egyed, W. (2011). Dose optimization in HDR brachytherapy: A literature review of quantitative models. Working Papers 2011/32. Hogeschool-Universiteit Brussel, Faculteit Economie en Management.

[15] Deasy, J. O. (1997). Multiple local minima in radiotherapy optimization problems with dose-volume constraints. Medical Physics, 24(7):1157-1161.

[16] Degerfält, J., Moegelin, I.-M., and Sharp, L. (2008). Strålbehandling. Second edition. Studentlitteratur, Lund.

[17] Ehrgott, M., Güler, Ç., Hamacher, H., and Shao, L. (2010). Mathematical optimization in intensity modulated radiation therapy. Annals of Operations Research, 175(1):309-365.

[18] Gabriel, J. (2007). The Biology of Cancer. Second edition. John Wiley \& Sons, Chichester.

[19] Garcia, M., Jemal, A., Ward, E. M., Center, M. M., Hao, Y., Siegel, R. L., and Thun, M. J. (2007). Global Cancer Facts and Figures 2007. American Cancer Society, Atlanta.

[20] Giantsoudi, D., Baltas, D., Karabis, A., Mavroidis, P., Zamboglou, N., Tselis, N., Shi, C., and Papanikolaou, N. (2013). A gEUD-based inverse planning technique for HDR prostate brachytherapy: Feasibility study. Medical Physics, 40(4):041704.

[21] Gorissen, B. L., den Hertog, D., and Hoffmann, A. L. (2013). Mixed integer programming improves comprehensibility and plan quality in inverse optimization of prostate HDR brachytherapy. Physics in Medicine and Biology, 58(4):1041-1057.

[22] Holder, A. (2004). Radiotherapy treatment design and linear programming. In Brandeau, M. L., Sainfort, F., and Pierskalla, W. P., Operations research and health care - A handbook of Methods and applications. Kluwer Academic Publishers, Norwell. 
[23] Hoskin, P. and Coyle, C. (2005). Radiotherapy in Practice: Brachytherapy. Oxford University Press, Oxford.

[24] Hoskin, P. J., Colombo, A., Henry, A., Niehoff, P., Hellebust, T. P., Siebert, F.-A., and Kovacs, G. (2013). GEC/ESTRO recommendations on high dose rate afterloading brachytherapy for localised prostate cancer: An update. Radiotherapy and Oncology, 107(3):325 - 332.

[25] Hsu, I.-C., Shinohara, K., Pouliot, J., Purdy, J., Michalski, J., and Ibbott, G. S. (2006). RTOG 0321 protocol: Phase II trial of combined high dose rate brachytherapy and external beam radiotherapy for adenocarcinoma of the prostate. Radiation Therapy Oncology Group.

[26] Joiner, M. and van der Kogel, A. (2009). Basic Clinical Radiobiology. Fourth edition. Hodder Arnold, London.

[27] Jozsef, G., Streeter, O., and Astrahan, M. (2003). The use of linear programming in optimization of HDR implant dose distributions. Medical Physics, 30(5):751-760.

[28] Karabis, A., Belotti, P., and Baltas, D. (2009). Optimization of catheter position and dwell time in prostate HDR brachytherapy using HIPO and linear programming. IFMBE Proceedings 25(1): 612-615.

[29] Kovács, G., Pötter, R., Loch, T., Hammer, J., Kolkman-Deurloo, I.-K., de la Rosette, J. J., and Bertermann, H. (2005). GEC/ESTRO-EAU recommendations on temporary brachytherapy using stepping sources for localised prostate cancer. Radiotherapy and Oncology, 74(2):137 - 148 .

[30] Lahanas, M., Baltas, D., Giannouli, S., Milickovic, N., and Zamboglou, N. (2000). Generation of uniformly distributed dose points for anatomy-based three-dimensional dose optimization methods in brachytherapy. Medical Physics, 27(5):10341046.

[31] Lahanas, M., Baltas, D., and Zamboglou, N. (2003). A hybrid evolutionary algorithm for multi-objective anatomy-based dose optimization in high-dose-rate brachytherapy. Physics in Medicine and Biology, 48(3):399-415.

[32] Lessard, E. and Pouliot, J. (2001). Inverse planning anatomy-based dose optimization for HDR-brachytherapy of the prostate using fast simulated annealing algorithm and dedicated objective function. Medical Physics, 28(5):773-779.

[33] Mavroidis, P., Katsilieri, Z., Kefala, V., Milickovic, N., Papanikolaou, N., Karabis, A., Zamboglou, N., and Baltas , D. (2010). Radiobiological evaluation of the influence of dwell time modulation restriction in HIPO optimized HDR prostate brachytherapy implants. Journal of Contemporary Brachytherapy, 2(3): 117-128.

[34] Milickovic, N., Lahanas, M., Papagiannopoulou, M., Zamboglou, N., and Baltas D. (2002). Multiobjective anatomy-based dose optimization for HDR-brachytherapy with constraint free deterministic algorithms. Physics in Medicine and Biology, 47(13):2263-2280. 
[35] Mould, R. F., Battermann, J. J., Martinez, A. A., and Speiser, B. L. (1994). Brachytherapy from radium to optimization. Nucletron BV, Veenendaal.

[36] National Cancer Institute (2010). Radiation therapy for cancer. http: / / www . cancer.gov/cancertopics/fact sheet/Therapy/radiation.

[37] Niemierko, A. (1997). Reporting and analyzing dose distributions: A concept of equivalent uniform dose. Medical Physics, 24(1):103-110.

[38] Niemierko, A. (1999). A generalized concept of equivalent uniform dose (EUD). Medical Physics, 26(6):1100.

[39] Ouhib, Z. (2005). HDR brachytherapy for prostate. In Thomadsen, B. R., Rivard, M. J., and Butler, W. M., Brachytherapy physics. Second edition. Medical Physics Publishing, Madison.

[40] Picard, J. C., Golshayan, A.-R., Marshall, D. T., Opfermann, K. J., and Keane, T. E. (2012). The multi-disciplinary management of high-risk prostate cancer. Urologic Oncology: Seminars and Original Investigations, 30(1):3-15.

[41] Prabhakar, R. (2010). Dose volume uniformity index: a simple tool for treatment plan evaluation in brachytherapy. Journal of Contemporary Brachytherapy, 2(2):7175 .

[42] Rivard, M. J., Coursey, B. M., DeWerd, L. A., Hanson, W. F., Huq, M. S., Ibbott, G. S., Mitch, M. G., Nath, R., and Williamson, J. F. (2004). Update of AAPM task group no. 43 report: A revised AAPM protocol for brachytherapy dose calculations. Medical Physics, 31(3):633-674.

[43] Rivard, M. J., Davis, S. D., DeWerd, L. A., Rusch, T. W., and Axelrod, S. (2006). Calculated and measured brachytherapy dosimetry parameters in water for the Xoft Axxent X-Ray Source: An electronic brachytherapy source. Medical Physics, 33(11):4020-4032.

[44] Rockafellar, R. and Uryasev, S. (2002). Conditional value-at-risk for general loss distributions. Journal of Banking \& Finance, 26(7):1443-1471.

[45] Romeijn, H. E., Ahuja, R. K., Dempsey, J. F., Kumar, A., and Li, J. G. (2003). A novel linear programming approach to fluence map optimization for intensity modulated radiation therapy treatment planning. Physics in Medicine and Biology, 48(21), 3521-3542.

[46] Ruotsalainen, H. (2009). Interactive Multiobjective Optimization in Model-based Decision Making with Applications. PhD thesis, University of Kuopio.

[47] Ruotsalainen, H., Miettinen, K., Palmgren, J.-E., and Lahtinen, T. (2010). Interactive multiobjective optimization for anatomy-based three-dimensional HDR brachytherapy. Physics in Medicine and Biology, 55(16):4703.

[48] Russell, K. R., Tedgren, A. K. C., and Ahnesjö, A. (2005). Brachytherapy source characterization for improved dose calculations using primary and scatter dose separation. Medical Physics, 32(9):2739-2752. 
[49] Saw, C. B., Suntharalingam, N., and Wu, A. (1993). Concept of dose nonuniformity in interstitial brachytherapy. International Journal of Radiation Oncology*Biology*Physics, 26(3):519-527.

[50] Shepard, D. M., Ferris, M. C., Olivera, G. H., and Mackie, T. R. (1999). Optimizing the delivery of radiation therapy to cancer patients. SIAM Review, 41(4):721-744.

[51] Siauw, T., Cunha, A., Atamturk, A., Hsu, I.-C., Pouliot, J., and Goldberg, K. (2011). IPIP: A new approach to inverse planning for HDR brachytherapy by directly optimizing dosimetric indices. Medical Physics, 38(7):4045-4051.

[52] Siauw, T., Cunha, A., Berenson, D., Atamtürk, A., Hsu, I.-C., Goldberg, K., and Pouliot, J. (2012). NPIP: A skew line needle configuration optimization system for HDR brachytherapy. Medical Physics, 39(7):4339-4346.

[53] Statens beredning för utvärdering av medicinsk metodik (2003). Strålbehandling vid cancer: En systematisk litteratur översikt. Statens beredning för utvärdering av medicinsk metodik (SBU), Stockholm.

[54] van't Riet, A., Mak, A. C., Moerland, M. A., Elders, L. H., and van der Zee, W. (1997). A conformation number to quantify the degree of conformality in brachytherapy and external beam irradiation: Application to the prostate. International Journal of Radiation Oncology*Biology*Physics, 37(3):731-736.

[55] Wu, A., Ulin, K., and Sternick, E. (1988). A dose homogeneity index for evaluating ${ }^{192}$ Ir interstitial breast implants. Medical Physics, 15(1):104-107.

[56] Wu, Q., Mohan, R., Niemierko, A., and Schmidt-Ullrich, R. (2002). Optimization of intensity-modulated radiotherapy plans based on the equivalent uniform dose. International Journal of Radiation Oncology*Biology*Physics, 52(1):224-235.

[57] Yamada, Y., Rogers, L., Demanes, D. J., Morton, G., Prestidge, B. R., Pouliot, J., Cohen, G. N., Zaider, M., Ghilezan, M., and Hsu, I.-C. (2012). American brachytherapy society consensus guidelines for high-dose-rate prostate brachytherapy. Brachytherapy, 11(1):20-32.

[58] Zinchenko, Y., Craig, T., Keller, H., Terlaky, T., and Sharpe, M. (2008). Controlling the dose distribution with gEUD-type constraints within the convex radiotherapy optimization framework. Physics in Medicine and Biology, 53(12):3231-3250. 


\section{Part II}

\section{Appended Papers}

The articles associated with this thesis have been removed for copyright reasons. For more details about these see:

http://urn.kb.se/resolve?urn=urn:nbn:se:liu:diva-99795 